\title{
ANALOGUES ELLIPTIQUES DES NOMBRES MULTIZÉTAS
}

\author{
BENJAMIN ENRIQUEZ
}

\begin{abstract}
RÉSumÉ. Nous étudions des fonctions du paramètre elliptique définies commes intégrales itérées de fonctions elliptiques. Nous établissons leur lien avec les "associateurs elliptiques" de notre précédent travail au moyen de réalisations fonctionnelles d'algèbres de Lie apparaissant dans cette théorie.
\end{abstract}

\section{INTRODUCTION}

La théorie des nombres multizétas a débuté dans [Za] avec la construction de familles de relations entre ces nombres, reposant en partie sur le lien observé par Kontsevich entre multizétas et intégrales itérées sur les espaces de modules de courbes rationnelles avec points marqués. Parallèlement, Drinfeld a établi des relations d'origine géométrique satisfaites par une série non-commutative, l'associateur KZ ([Dr] ) ; Le et Murakami ont identifié l'associateur KZ à une série génératrice des multizétas ([LM]), ce qui a permis de considérer les relations de l'associateur $\mathrm{KZ}$ comme un deuxième système de relations entre multizétas. Le lien entre les deux systèmes de relations a été étudié par Furusho $([\mathrm{Fu}])$.

Un analogue elliptique de la théorie des associateurs a été construit dans [En2] à partir d'un analogue elliptique de la connection de Knizhnik-Zamolodchikov ([CEE], voir aussi [LR]). Le rôle de l'associateur KZ y est tenu par un couple de fonctions $(A(\tau), B(\tau))$ d'un paramètre $\tau$ dans le demi-plan de Poincaré, à valeurs dans un groupe de séries non-commutatives à deux variables $\exp \left(\hat{\mathfrak{f}}_{2}\right)$. Les résultats principaux de [En2] sur le couple $(A(\tau), B(\tau))$ sont : le comportement de ce couple sous les transformations modulaires ; une famille de relations algébriques (d'origine géométrique) satisfaites par $(A(\tau), B(\tau))$; une équation différentielle satisfaite par le même objet ; son comportement en $\tau \rightarrow \mathrm{i} \infty$. Un corollaire de cette étude est une famille de relations algébriques entre intégrales itérées de séries d'Eisenstein et multizétas. Un rôle important est joué dans cette théorie, et également dans la théorie reliée des motifs elliptiques universels $([\mathrm{HM}, \overline{\mathrm{Pk}}])$, par une algèbre de Lie $\left\langle\delta_{2 n}, n \geq-1\right\rangle \subset \operatorname{Der}\left(\mathfrak{f}_{2}\right)$. Nous rappelons ces résultats en section 1 .

Le but principal de cet article est l'étude des coefficients des séries $A(\tau), B(\tau)$. Il s'agit de fonctions

$$
I_{\underline{d}}(\tau), J_{\underline{d}}(\tau), \quad \underline{d}=\left(d_{1}, \ldots, d_{n}\right) \in\{-1,0,1, \ldots\}^{n}
$$

du paramètre elliptique, qui sont des analogues elliptiques des nombres multizétas.

La section 2 est consacrée à la détermination d'expressions intégrales pour ces fonctions. Nous utilisons pour cela le calcul de l'holonomie régularisée des équations différentielles sur ]0,1[ à valeurs dans une algèbre libre, avec singularités aux extrémités. Ce calcul a été effectué dans [En1] à partir d'idées contenues dans [LM]. Le résultat de [En1] est formulé en sections 2.1 et 2.2, et appliqué en sections 2.3 et 2.4 au calcul d'expressions intégrales pour les $I_{\underline{d}}(\tau), J_{\underline{d}}(\tau)$ (relations (18), (19), (20)), (21)). En section 2.5, nous traduisons en termes des $I_{\underline{d}}(\tau), \underline{J_{\underline{d}}}(\tau)$ certaines identités satisfaites par $(A(\tau), B(\tau))$.

La section 3 est consacrée aux systèmes différentiels satisfaits par les $I_{\underline{d}}(\tau), J_{\underline{d}}(\tau)$. En section 3.1, on construit des systèmes différentiels satisfaits par des intégrales itérées générales du type de celles introduites en section 2.2. En section 3.2, on applique ce résultat aux fonctions $I_{\underline{d}}(\tau), J_{\underline{d}}(\tau)$ et on obtient ainsi un système différentiel satisfait par ces fonctions (théorème 3.10). 
En section 4, on montre l'équivalence entre ce système différentiel et le système différentiel satisfait par $(A(\tau), B(\tau))$ explicité dans En2. Ceci est réalisé au moyen d'une réalisation fonctionnelle de l'algèbre de Lie $\left\langle\delta_{2 n}, n \geq-1\right\rangle$ (section 4).

Enfin, en section 5 , on applique le système différentiel du théorème 3.10 au développement asymptotique des $I_{\underline{d}}(\tau), J_{\underline{d}}(\tau)$ en $\tau \rightarrow \mathrm{i} \infty$; on montre que ce développement asymptotique s'exprime à l'aide de nombres multizétas.

Signalons enfin les liens possibles entre le présent travail et BL : les auteurs de cet article construisent une théorie des polylogarithmes elliptiques multiples, qui sont certaines fonctions multivaluées sur la variété $E_{\tau}^{n}$, où $E_{\tau}:=\mathbb{C} /(\mathbb{Z}+\tau \mathbb{Z})$. Ils projettent d'en déduire, par spécialisation, des fonctions de $\tau$ qu'ils appellent "fonctions multizétas elliptiques". On peut s'attendre à ce que ces fonctions présentent des liens étroits avec les fonctions $I_{\underline{d}}(\tau), J_{\underline{d}}(\tau)$ du présent article.

\section{Préliminaires : associateurs elliptiques}

Dans cette section, nous rappelons la construction et les propriétés de la fonction $\tau \mapsto(A(\tau), B(\tau))$ $([\mathrm{En} 2])$.

1.1. Définition de $(A(\tau), B(\tau))$. Soit pour $n \geq 2, \overline{\mathfrak{t}}_{1, n}$ l'algèbre de Lie présentée par les générateurs $x_{i}, y_{i}, i \in\{1, \ldots, n\}$ et les relations $\sum_{i} x_{i}=\sum_{i} y_{i}=0,\left[x_{i}, x_{j}\right]=\left[y_{i}, y_{j}\right]=0,\left[x_{i}, y_{j}\right]=\left[x_{j}, y_{i}\right]=: t_{i j}$ si $i \neq j,\left[x_{k}, t_{i j}\right]=\left[y_{k}, t_{i j}\right]=0$ si $i, j, k$ sont distincts. En particulier, l'algèbre de Lie $\overline{\mathfrak{t}}_{1,2}$ s'identifie à l'algèbre de Lie $\mathfrak{f}_{2}$ librement engendrée par les deux générateurs $x:=x_{1}$ et $y:=y_{1}$.

Soit $\mathfrak{H}:=\{\tau \in \mathbb{C} \mid \Im(\tau)>0\}$ le demi-plan de Poincaré. On note $(z, \tau) \mapsto \theta_{\tau}(z)$ la fonction sur $\mathbb{C} \times \mathfrak{H}$ donnée par 1

$$
\theta_{\tau}(z):=\frac{e^{\pi \mathrm{i} z}-e^{-\pi \mathrm{i} z}}{2 \pi \mathrm{i}} \prod_{n>0} \frac{\left(1-e^{2 \pi \mathrm{i}(z+n \tau)}\right)\left(1-e^{2 \pi \mathrm{i}(-z+n \tau)}\right)}{\left(1-e^{2 \pi \mathrm{i} n \tau}\right)^{2}} .
$$

On a $\theta_{\tau}(z+1)=-\theta_{\tau}(z)=\theta_{\tau}(-z), \theta_{\tau}(z+\tau)=-e^{-\mathrm{i} \pi \tau} e^{-2 \pi \mathrm{i} z} \theta_{\tau}(z), \frac{\partial}{\partial z} \theta_{\tau}(z)_{\mid z=0}=1$, et $\left(\theta_{\tau}(-)\right)^{-1}(0)=$ $\mathbb{Z}+\tau \mathbb{Z}$. La fonction $\theta_{\tau}(-)$ est reliée à la fonction thêta de Jacobi donnée par

$$
\vartheta_{1}(z, \tau)=-\sum_{n \in \mathbb{Z}+\frac{1}{2}} e^{\pi \mathrm{i} n^{2}+2 \pi \mathrm{i} n\left(z+\frac{1}{2}\right)}
$$

par l'identité $\vartheta_{1}(z, \tau)=2 \pi \eta(\tau)^{3} \theta_{\tau}(z)$, où $\eta(\tau)=q^{\frac{1}{24}} \prod_{n>0}\left(1-q^{n}\right)$ et $q=e^{2 \pi \mathrm{i} \tau}$.

On définit $A(\tau), B(\tau)$ comme les holonomies régularisées de l'équation différentielle

$$
X^{\prime}(z)=-\frac{\theta_{\tau}(z+\operatorname{ad} x) \operatorname{ad} x}{\theta_{\tau}(z) \theta_{\tau}(\operatorname{ad} x)}(y) \cdot X(z)
$$

à valeurs dans le groupe $\exp \left(\hat{\overline{\mathfrak{t}}}_{1,2}\right)$ le long les chemins $[0,1]$ et $[0, \tau]$ (l'algèbre de Lie est complétée pour le degré en $x, y)$. Dans cette équation, on donne à l'expression $f(z, \operatorname{ad} x)(y)$ la valeur $\sum_{n \geq 0} g_{n}(z)(\operatorname{ad} x)^{n}(y)$, où $g_{n}(z):=\frac{1}{n !}\left(\frac{\partial}{\partial t}\right)^{n} f(z, t)_{\mid t=0}$, et $f(z, t)$ est une fonction analytique en deux variables, régulière en $t=0$ (ici $f(z, t)=-\frac{\theta_{\tau}(z+t)}{\theta_{\tau}(z)} \frac{t}{\theta_{\tau}(t)}$, qui vaut -1 en $t=0$ ). Cette équation admet une solution $X(z)$ définie sur $\{a+b \tau \mid(a, b) \in] 0,1\left[^{2}\right\}$ telle que $X(z) \simeq(-2 \pi \mathrm{i} z)^{-[x, y]}$ en $z \rightarrow 0$. Alors

$$
A(\tau):=X(z)^{-1} X(z+1), \quad B(\tau):=X(z)^{-1} e^{2 \pi \mathrm{i} x} X(z+\tau)
$$

Ce sont des éléments du groupe $\exp \left(\hat{\overline{\mathfrak{t}}}_{1,2}\right)$.

\subsection{Propriétés de $A(\tau), B(\tau)$.}

$\overline{1_{\text {On note } \mathrm{i}:=}} \sqrt{-1}$. 
1.2.1. Propriétés modulaires. On a d'après [En2], Proposition 66

$$
A\left(\frac{-1}{\tau}\right)=\operatorname{Ad}\left(\frac{-1}{\tau}\right)^{-t} \circ \alpha_{\tau}\left(B(\tau)^{-1}\right), \quad B\left(\frac{-1}{\tau}\right)=\operatorname{Ad}\left(\frac{-1}{\tau}\right)^{-1} \circ \alpha_{\tau}\left(B A B^{-1}(\tau)\right),
$$

où $\alpha_{\tau} \in \operatorname{Aut}\left(\exp \left(\hat{\mathfrak{f}}_{2}\right)\right)$ est donné par $x \mapsto-\tau x, y \mapsto-2 \pi \mathrm{i} x-\tau^{-1} y$,

$$
t:=-[x, y]
$$

et $\left(-\tau^{-1}\right)^{-t}:=\exp \left(-\log \left(-\tau^{-1}\right) t\right)$, la détermination du logarithme étant de partie imaginaire comprise entre 0 et $\pi$.

Les identités $\left(-I_{2}\right)(A(\tau))=A(\tau)^{2,1},\left(-I_{2}\right)(B(\tau))=B(\tau)^{2,1}$, dans lesquelles $\left(-I_{2}\right)$ est l'automorphisme de $\exp \left(\hat{\mathfrak{f}}_{2}\right)$ induit par $x \mapsto-x, y \mapsto-y$, et les identités (5) (voir section 1.2.2) impliquent

$$
\left(-I_{2}\right)(A(\tau))=e^{-\mathrm{i} \pi t} A(\tau)^{-1} e^{-\mathrm{i} \pi t}, \quad\left(-I_{2}\right)(B(\tau))=e^{\mathrm{i} \pi t} B(\tau)^{-1} e^{\mathrm{i} \pi t} .
$$

Compte tenu des ces identités et de (6), les deux parties de (2) sont équivalentes après application de $\tau \mapsto-\tau^{-1}$ à l'une d'elles.

1.2.2. Relations algébriques. Soit $\Phi(a, b)$ l'associateur KZ, défini par $\Phi(a, b)=Y_{1}^{-1} Y_{0}$, où $Y_{i}$ sont les solutions de $Y^{\prime}(z)=(a / z+b /(z-1)) Y(z)$ sur $] 0,1\left[\right.$ telles que $Y_{0}(z) \simeq z^{a}$ en $z \rightarrow 0, Y_{1}(z) \simeq(1-z)^{b}$ en $z \rightarrow 1$, et où $a, b$ sont des variables formelles non-commutatives.

On pose

$$
\alpha_{+}:=e^{\mathrm{i} \pi\left(t_{12}+t_{13}\right)} A(\tau)^{1,23} \Phi\left(t_{12}, t_{23}\right), \quad \alpha_{-}:=e^{-\mathrm{i} \pi\left(t_{12}+t_{13}\right)} B(\tau)^{1,23} \Phi\left(t_{12}, t_{23}\right),
$$

où $a \mapsto a^{1,23}$ est le morphisme d'algèbres de Lie $\overline{\mathfrak{t}}_{1,2} \rightarrow \overline{\mathfrak{t}}_{1,3}$ tel que $x_{1} \mapsto x_{1}, x_{2} \mapsto x_{2}+x_{3}, y_{1} \mapsto y_{1}$, $y_{2} \mapsto y_{2}+y_{3}$.

La première famille de relations satisfaites par $(A(\tau), B(\tau))$ est

$$
\alpha_{ \pm}^{3,1,2} \alpha_{ \pm}^{2,3,1} \alpha_{ \pm}=1 \quad\left(\text { dans } \exp \left(\hat{\overline{\mathfrak{t}}}_{1,3}\right)\right)
$$

où $a \mapsto a^{2,3,1}$ est l'automorphisme de $\overline{\mathfrak{t}}_{1,3}$ tel que $x_{i} \mapsto x_{i+1} \bmod 3, y_{i} \mapsto y_{i+1} \bmod 3$, et $a \mapsto a^{3,1,2}$ est le carré de cet automorphisme.

Le couple $(A(\tau), B(\tau))$ satisfait d'autre part la relation

$$
\left(\Phi\left(t_{12}, t_{23}\right)^{-1} * B(\tau)^{1,23},\left(e^{-\mathrm{i} \pi t_{12}} \Phi\left(t_{21}, t_{13}\right)\right) *\left(A(\tau)^{2,13}\right)^{-1}\right)=e^{2 \pi \mathrm{i} t_{12}} \quad\left(\text { dans } \exp \left(\hat{\overline{\mathfrak{t}}}_{1,3}\right)\right),
$$

où $(x, y):=x y x^{-1} y^{-1}, x * y:=x y x^{-1}, t_{12}:=\left[x_{1}, y_{2}\right]$ et $a \mapsto a^{2,13}$ est le morphisme $\overline{\mathfrak{t}}_{1,2} \rightarrow \overline{\mathfrak{t}}_{1,3}$ donné par $x_{1} \mapsto x_{2}, x_{2} \mapsto x_{1}+x_{3}, y_{1} \mapsto y_{2}, y_{2} \mapsto y_{1}+y_{3}$.

Les relations (3) impliquent alors

$$
e^{\mathrm{i} \pi t} A(\tau) e^{\mathrm{i} \pi t} A(\tau)^{2,1}=e^{-\mathrm{i} \pi t} B(\tau) e^{-\mathrm{i} \pi t} B(\tau)^{2,1}=1 \quad\left(\text { dans } \exp \left(\hat{\overline{\mathfrak{t}}}_{1,2}\right)\right),
$$

où $a \mapsto a^{2,1}$ est l'automorphisme involutif de $\overline{\mathfrak{t}}_{1,2}$ donné par $x_{1} \leftrightarrow x_{2}, y_{1} \leftrightarrow y_{2}$, et la relation (44) implique

$$
(A(\tau), B(\tau))=e^{-2 \pi \mathrm{i} t} \quad\left(\text { dans } \exp \left(\hat{\overline{\mathfrak{t}}}_{1,2}\right)\right)
$$

1.2.3. Equations différentielles. Pour chaque $n \geq-1$, il existe une unique dérivation de $\mathfrak{f}_{2}$, homogène pour le bidegré en $(x, y)$ et telle que $\delta_{2 n}(x)=\operatorname{ad}(x)^{2 n+2}(y)=:\left[x^{2 n+2} y\right]$ et $\delta_{2 n}([x, y])=0$. Les fonctions $A(\tau), B(\tau)$ satisfont alors les équations différentielles

$2 \pi \mathrm{i} \partial_{\tau} A(\tau)=-\left(\sum_{n \geq-1}(2 n+1) G_{2 n+2}(\tau) \delta_{2 n}\right)(A(\tau)), \quad 2 \pi \mathrm{i} \partial_{\tau} B(\tau)=-\left(\sum_{n \geq-1}(2 n+1) G_{2 n+2}(\tau) \delta_{2 n}\right)(B(\tau))$.

(cf. [En2, Proposition 67), où les séries d'Eisenstein sont définies par

$$
G_{k}(\tau)=\sum_{a \in(\mathbb{Z}+\tau \mathbb{Z})-\{0\}} \frac{1}{a^{k}} \quad \text { si } k \text { est pair } \geq 4, \quad G_{2}(\tau)=\sum_{m \in \mathbb{Z}}\left(\sum_{n}^{\prime} \frac{1}{(n+m \tau)^{2}}\right), \quad G_{0}(\tau):=-1,
$$


et $\sum^{\prime}$ signifie $\sum_{n \in \mathbb{Z}}$ si $m \neq 0$ et $\sum_{n \in \mathbb{Z}-\{0\}}$ si $m=0$.

1.2.4. Comportement à l'infini. On a

$$
\begin{gathered}
A(\tau)=\Phi(\tilde{y}, t) e^{2 \pi \mathrm{i} \tilde{y}} \Phi(\tilde{y}, t)^{-1}+O\left(e^{2 \pi \mathrm{i} \tau}\right), \\
B(\tau)=e^{\mathrm{i} \pi t} \Phi(-\tilde{y}-t, t) e^{2 \pi \mathrm{i} x} e^{2 \pi \mathrm{i} \tilde{y} \tau} \Phi(\tilde{y}, t)^{-1}+O\left(e^{2 \pi \mathrm{i}(1-\epsilon) \tau}\right)
\end{gathered}
$$

pour tout $\epsilon>0$, lorsque $\tau \rightarrow \mathrm{i} \infty$ ([CEE], démonstration de Proposition 4.7 puis Lemma 4.14), où

$$
\tilde{y}:=-\frac{\operatorname{ad} x}{e^{2 \pi \operatorname{iad} x}-1}(y)
$$

et on rappelle que $t=-[x, y]$ et $\Phi(a, b)$ est l'associateur KZ défini en section 1.2 .2 ,

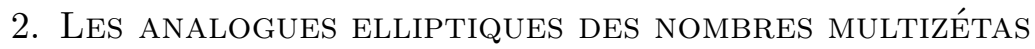

Dans cette section, nous calculons l'holonomie régularisée de certaines équations différentielles (section 2.1). Nous exprimons cette holonomie en termes d'intégrales itérées régularisées (section 2.21). Nous utilisons ces régularisations en section 2.3 pour définir les fonctions du paramètre elliptique, analogues des nombres multizétas. Nous montrons que les fonctions $A(\tau), B(\tau)$ peuvent s'interpréter comme des séries génératrices pour ces fonctions (section 2.4). Les propriétés de $A(\tau), B(\tau)$ peuvent donc se traduire en termes fonctionnels : c'est ce qui est fait explicitement en section 2.5 pour certaines de ces propriétés.

2.1. Holonomies régularisées. Soit $I$ un ensemble fini contenant les éléments 0,1 . Soit $\Omega^{1}:=$ $\Omega^{1}(] 0,1[, \mathbb{C})$ l'espace des formes différentielles sur $] 0,1\left[\right.$ et soit $i \mapsto \underline{\omega}_{i}$ une application $I \rightarrow \Omega^{1}$, telle que $\underline{\omega}_{0}=d \log (z), \underline{\omega}_{1}=d \log (1-z)$, et pour $i \neq 0,1$, la forme $\underline{\omega}_{i}$ est régulière en 0 et 1 .

Soit $V:=\oplus_{i \in I} \mathbb{C} a_{i}$ l'espace vectoriel engendré par $I$. On pose

$$
\omega:=\sum_{i \in I} \underline{\omega}_{i} \cdot a_{i} \in \Omega^{1}(] 0,1[, V) .
$$

On note $T(V)$ l'algèbre tensorielle de $V$, munie du produit de concaténation, et $\hat{T}(V)$ sa complétion pour le degré pour lequel $V$ est de degré 1 . L'équation différentielle $d f=\omega f$ d'inconnue une fonction $f:] 0,1\left[\rightarrow \hat{T}(V)\right.$ admet deux solutions $f_{0}$ et $f_{1}$, telles que $f_{0}(z) \sim z^{a_{0}}$ pour $z \rightarrow 0$ et $f_{1}(z) \sim(1-z)^{a_{1}}$ pour $z \rightarrow 1$. On définit alors l'holonomie régularisée de cette équation différentielle comme

$$
\operatorname{Hol}([0,1], \omega):=f_{1}^{-1} f_{0} \in \hat{T}(V) .
$$

D'après [En1], appendice, on a

$$
\begin{aligned}
& \operatorname{Hol}([0,1], \omega) \\
& =1+\sum_{n \geq 1} \sum_{\substack{\left(i_{1}, \ldots, i_{n}\right) \in I^{n}, i_{1} \neq 0, i_{n} \neq 1}}\left(\int_{[0,1]} \underline{\omega}_{i_{1}} \cdots \cdots \underline{\omega}_{i_{n}}\right) \sum_{\substack{A \subset\left\{a \mid i_{a}=0\right\}, B \subset\left\{b \mid i_{b}=1\right\}}}\left(-a_{1}\right)^{|B|}\left(a_{i_{n}} \cdots a_{i_{1}}\right)_{A, B}\left(-a_{0}\right)^{|A|},
\end{aligned}
$$

où $\left(a_{i_{n}} \cdots a_{i_{1}}\right)_{A, B}$ est le produit des $a_{i_{k}}$, dans lequel $k$ parcourt de facon décroissante l'ensemble $[1, n]-(A \cup B)$, et où on pose $\int_{[0,1]} \alpha_{1} \cdots \bullet \alpha_{n}:=\int_{\Delta_{n}} \alpha_{1} \otimes \cdots \otimes \alpha_{n}$, où $\Delta_{n}$ est le simplexe $\left\{\left(t_{1}, \ldots, t_{n}\right) \in \mathbb{R}^{n} \mid 0 \leq t_{1} \leq \ldots \leq t_{n} \leq 1\right\}$ pour $\alpha_{1}, \ldots, \alpha_{n} \in \Omega^{1}$.

Soit $\operatorname{Hol}_{n}([0,1], \omega)$ la partie de degré $n$ de cette somme. Alors

$$
\operatorname{Hol}([0,1], \omega)=\sum_{n \geq 0} \operatorname{Hol}_{n}([0,1], \omega) .
$$

Soit $x \mapsto x^{o p}$ l'involution de $T(V)$ donnée par $\left(v_{1} \otimes \cdots \otimes v_{k}\right)^{o p}:=v_{k} \otimes \cdots \otimes v_{1}$. Soit $m: \mathbb{C}\left[a_{0}\right] \otimes$ $T(V) \otimes \mathbb{C}\left[a_{1}\right] \rightarrow T(V)$ l'application composée $\mathbb{C}\left[a_{0}\right] \otimes T(V) \otimes \mathbb{C}\left[a_{1}\right] \hookrightarrow T(V)^{\otimes 3} \rightarrow T(V)$, où 
la première application est un produit tensoriel d'injections canoniques et de l'identité, et où la deuxième application est la multiplication de $T(V)$. Soit $O p$ l'endomorphisme de $T(V)$ donné par

$$
O p=\left(T(V) \stackrel{\text { mor }}{\rightarrow} \mathbb{C}\left[a_{0}\right] \otimes T(V) \otimes \mathbb{C}\left[a_{1}\right] \stackrel{m}{\rightarrow} T(V)\right)
$$

où mor est le morphisme d'algèbres induit par $a_{0} \mapsto a_{0}^{(2)}-a_{0}^{(1)}, a_{1} \mapsto a_{1}^{(2)}-a_{1}^{(3)}, a_{i} \mapsto a_{i}^{(2)}, i \neq 0$ (on note $a_{0}^{(1)}:=a_{0} \otimes 1 \otimes 1, a_{i}^{(2)}:=1 \otimes a_{i} \otimes 1, a_{1}^{(3)}:=1 \otimes 1 \otimes a_{1}$ ).

Posons $\omega_{0}:=\underline{\omega}_{0} \otimes a_{0}, \omega_{1}:=\underline{\omega}_{1} \otimes a_{1} ;$ ce sont des éléments de $\Omega^{1} \otimes V$. Alors

$$
\operatorname{Hol}_{0}([0,1], \omega)=1, \quad \operatorname{Hol}_{1}([0,1], \omega)=\left(\int_{[0,1]} \otimes \mathrm{id}\right)\left(\omega-\omega_{0}-\omega_{1}\right),
$$

et si $n \geq 2$,

$$
H_{o l}([0,1], \omega)^{o p}=\left(\int_{\Delta_{n}} \otimes O p\right)\left(\left(\omega-\omega_{0}\right) \circ \omega^{\circ n-2} \circ\left(\omega-\omega_{1}\right)\right)
$$

où $\circ$ est le produit de l'algèbre tensorielle $T\left(\Omega^{1} \otimes V\right)$.

Notons $\omega \mapsto \omega^{01}, \omega^{02}, \omega^{03}$ les applications naturelles de $\Omega^{1} \otimes \mathbb{C} a_{0}, \Omega^{1} \otimes V, \Omega^{1} \otimes \mathbb{C} a_{1}$ vers $T\left(\Omega^{1}\right) \otimes$ $\mathbb{C}\left[a_{0}\right] \otimes T(V) \otimes \mathbb{C}\left[a_{1}\right]$. Alors (11) implique que pour $n \geq 2$,

$$
\operatorname{Hol}_{n}([0,1], \omega)^{o p}=\left(\int_{\Delta_{n}} \otimes m\right)\left(\left(\left(\omega-\omega_{0}\right)^{02}-\omega_{1}^{03}\right) \circ\left(\omega^{02}-\omega_{0}^{01}-\omega_{1}^{03}\right)^{\circ n-2} \circ\left(\left(\omega-\omega_{1}\right)^{02}-\omega_{0}^{01}\right)\right),
$$

où le terme entre crochets est un élément de $T\left(\Omega^{1}\right) \otimes \mathbb{C}\left[a_{0}\right] \otimes T(V) \otimes \mathbb{C}\left[a_{1}\right]$.

On montre facilement l'énoncé suivant :

Lemme 2.1. Soit $\Omega, V$ des espaces vectoriels, soit $A$ une algèbre commutative. On note $\omega \mapsto \omega^{12}$, $\alpha \mapsto \alpha^{13}$ les applications naturelles de $\Omega \otimes V$ et $\Omega \otimes A$ vers $T(\Omega) \otimes T(V) \otimes A$. On note $\amalg$ le produit de battage de $T(\Omega)$, donné par $\left(x_{1} \otimes \cdots \otimes x_{k}\right)\left(x_{k+1} \otimes \cdots \otimes x_{k+l}\right)=\sum x_{f(1)} \otimes \cdots \otimes x_{f(k+l)}$, où la somme parcourt les permutations de $[1, k+l]$ telles que si $f(i)<f(j) \leq k$ ou $l+1 \leq f(i)<f(j)$, alors $i<j$. On note également $\mathrm{m}:(T(\Omega) \otimes T(V)) \otimes(T(\Omega) \otimes A) \rightarrow T(\Omega) \otimes T(V) \otimes A$ le produit tensoriel du produit $\amalg: T(\Omega)^{\otimes 2} \rightarrow T(\Omega)$ avec les endomorphismes identité de $T(V)$ et de $A$.

Si $\omega \in \Omega \otimes V, \alpha \in \Omega \otimes A$, alors

$$
\left(\omega^{12}+\alpha^{13}\right)^{\circ n}=\sum_{k=0}^{n} \omega^{\circ n-k} \amalg \alpha^{\circ k},
$$

où les puissances sont calculées dans les produits tensoriels $T(\Omega) \otimes T(V) \otimes A, T(\Omega) \otimes T(V)$ et $T(\Omega) \otimes A$, où $T(\Omega)$ est muni du produit de battage et $T(V)$ du produit de concaténation.

En appliquant cette égalité au membre de droite de (12), on trouve

$$
\begin{aligned}
H o l_{n}([0,1], \omega)^{o p}=\sum_{\substack{k, l \geq 0 \\
k+l \leq n-2}}( & \left.-a_{0}\right)^{k}\left(\int_{\Delta_{n}}\left(\omega-\omega_{0}\right) \circ\left(\underline{\omega}_{0}^{\circ k} \amalg \underline{\omega}_{1}^{\circ l} \amalg \omega^{\circ n-2-k-l}\right) \circ\left(\omega-\omega_{1}\right)\right)\left(-a_{1}\right)^{l} \\
& +\left(-a_{0}\right)^{k}\left(\int_{\Delta_{n}} \underline{\omega}_{1} \circ\left(\underline{\omega}_{0}^{\circ k} \amalg \underline{\omega}_{1}^{\circ l} \amalg \omega^{\circ n-2-k-l}\right) \circ\left(\omega-\omega_{1}\right)\right)\left(-a_{1}\right)^{l+1} \\
& \left.+\left(-a_{0}\right)^{k+1}\left(\int_{\Delta_{n}}\left(\omega-\omega_{0}\right) \circ\left(\underline{\omega}_{0}^{\circ k} \amalg \underline{\omega}_{1}^{\circ l} \amalg \omega^{\circ n-2-k-l}\right) \circ \underline{\omega}_{0}\right)\right)\left(-a_{1}\right)^{l} \\
& \left.+\left(-a_{0}\right)^{k+1}\left(\int_{\Delta_{n}} \underline{\omega}_{1} \circ\left(\underline{\omega}_{0}^{\circ k} \amalg \underline{\omega}_{1}^{\circ l} \amalg \omega^{\circ n-2-k-l}\right) \circ \underline{\omega}_{0}\right)\right)\left(-a_{1}\right)^{l+1}
\end{aligned}
$$

pour $k \geq 2$, expression dans lequelle o est le produit de concaténation dans $T\left(\Omega^{1}\right)$ ou $T\left(\Omega^{1}\right) \otimes T(V)$, l'espace $T\left(\Omega^{1}\right)$ est considéré comme un sous-espace de $T\left(\Omega^{1}\right) \otimes T(V)$ par tensorisation avec 1 , le symbole $ш$ désigne le produit sur $T\left(\Omega^{1}\right) \otimes T(V)$, produit tensoriel du produit de battage et 
du produit de concaténation, et $\int_{\Delta_{n}}: T\left(\Omega^{1}\right) \otimes T(V) \rightarrow T(V)$ désigne le produit tensoriel de $\int_{\Delta_{n}}: T\left(\Omega^{1}\right) \rightarrow \mathbb{C}$ avec l'identité de $T(V)$.

En utilisant l'identité $(a \circ A) \amalg(b \circ B)=a \circ(A \amalg(b \circ B))+b \circ(B \amalg(a \circ A))$ dans $T\left(\Omega^{1}\right)$, où $a, b \in \Omega^{1}$, $A, B \in T\left(\Omega^{1}\right)$, on simplifie ainsi cette expression

$$
H o l_{n}([0,1], \omega)^{o p}=\int_{\Delta_{n}} \sum_{\substack{k, l \geq 0 \\ k+l \leq n-2}}\left(-a_{0}\right)^{k}\left(\underline{\omega}_{0}^{\circ k} \amalg \underline{\omega}_{1}^{\circ l} \amalg \omega^{\circ n-2-k-l}\right)\left(-a_{1}\right)^{l},
$$

dans laquelle les signes somme et intégrale ne peuvent être inversés, les termes individuels de la somme n'étant pas intégrables.

Remarque 2.2. On peut réduire la dimension du simplexe d'intégration dans les formules (13), (14) en utilisant l'identité

$$
\int_{\Delta_{n}} \alpha \circ\left((d f)^{\circ a} \amalg(d g)^{\circ b} \amalg \beta\right) \circ \gamma=\sum_{a^{\prime}, a^{\prime \prime} \mid a^{\prime}+a^{\prime \prime}=a} \sum_{b^{\prime}, b^{\prime \prime} \mid b^{\prime}+b^{\prime \prime}=b} \int_{\Delta_{n-a-b}} \frac{(-f)^{a^{\prime \prime}}}{a^{\prime \prime} !} \frac{(-g)^{b^{\prime \prime}}}{b^{\prime \prime} !} \alpha \circ \beta \circ \gamma \frac{f^{a^{\prime}}}{a^{\prime} !} \frac{g^{b^{\prime \prime}}}{b^{\prime \prime} !}
$$

dans laquelle $\alpha, \beta, \gamma$ sont dans $T\left(\Omega^{1}\right)$ et $f, g$ sont des fonctions sur $[0,1]$.

2.2. Intégrales itérées régularisées. Soit $\mathcal{A}$ une algèbre, limite projective d'algèbres de dimension finie. Soit $\mathbf{a}_{0}, \mathbf{a}_{1}$ des éléments de $\mathcal{A}$, et posons

$$
\Omega_{\mathbf{a}_{0}, \mathbf{a}_{1}}:=\left\{\boldsymbol{\omega} \in \Omega^{1}(] 0,1[, \mathcal{A}) \mid \boldsymbol{\omega}=\mathbf{a}_{0} d \log (z)+O(1) \text { si } z \rightarrow 0, \boldsymbol{\omega}=\mathbf{a}_{1} d \log (1-z)+O(1) \text { si } z \rightarrow 1\right\} .
$$

Pour $\boldsymbol{\omega} \in \Omega_{\mathbf{a}_{0}, \mathbf{a}_{1}}$, on pose

$$
I_{[0,1]}^{r e g}(\boldsymbol{\omega}):=\int_{[0,1]}\left(\boldsymbol{\omega}-\mathbf{a}_{0} d \log (z)-\mathbf{a}_{1} d \log (1-z)\right) .
$$

Pour $n \geq 2$ et $\boldsymbol{\omega}_{1}, \ldots, \boldsymbol{\omega}_{n} \in \Omega_{\mathbf{a}_{0}, \mathbf{a}_{1}}$, on pose

$$
I_{[0,1]}^{r e g}\left(\boldsymbol{\omega}_{1}, \ldots, \boldsymbol{\omega}_{n}\right):=\sum_{\substack{a, b, \epsilon, \eta \mid \\ a, b \geq 0, a+b \leq n-2, \epsilon, \eta \in\{0,1\}}} \mathbf{a}_{0}^{a+\epsilon} \cdot I_{a, b}^{\epsilon \eta} \cdot \mathbf{a}_{1}^{b+\eta},
$$

le produit étant calculé dans $\mathcal{A}^{o p}$ (l'algèbre opposée à $\mathcal{A}$ ), où

$$
\begin{aligned}
I_{a, b}^{00} & =\int_{\Delta_{n}}\left(\boldsymbol{\omega}_{a+1}-\mathbf{a}_{0} d \log (z)\right) \circ\left((-d \log (z))^{\circ a} \amalg(-d \log (1-z))^{\circ b} \amalg\left(\boldsymbol{\omega}_{a+2} \circ \cdots \circ \boldsymbol{\omega}_{n-b-1}\right)\right) \circ\left(\boldsymbol{\omega}_{n-b}-\mathbf{a}_{1} d \log (1-z)\right), \\
I_{a, b}^{01} & =\int_{\Delta_{n}}(-d \log (1-z)) \circ\left((-d \log (z))^{\circ a} \amalg(-d \log (1-z))^{\circ b} \amalg\left(\boldsymbol{\omega}_{a+1} \circ \cdots \circ \boldsymbol{\omega}_{n-b-2}\right)\right) \circ\left(\boldsymbol{\omega}_{n-b-1}-\mathbf{a}_{1} d \log (1-z)\right), \\
I_{a, b}^{10} & =\int_{\Delta_{n}}\left(\boldsymbol{\omega}_{a+2}-\mathbf{a}_{0} d \log (z)\right) \circ\left((-d \log (z))^{\circ a} \amalg(-d \log (1-z))^{\circ b} \amalg\left(\boldsymbol{\omega}_{a+3} \circ \cdots \circ \boldsymbol{\omega}_{n-b}\right)\right) \circ(-d \log (z)), \\
I_{a, b}^{11} & =\int_{\Delta_{n}}(-d \log (1-z)) \circ\left((-d \log (z))^{\circ a} \amalg(-d \log (1-z))^{\circ b} \amalg\left(\boldsymbol{\omega}_{a+2} \circ \cdots \circ \boldsymbol{\omega}_{n-b-1}\right)\right) \circ(-d \log (z)),
\end{aligned}
$$

où : o est le produit dans $T\left(\Omega^{1}\right) \otimes \mathcal{A}^{o p}$, les formes $d \log (z), d \log (1-z)$ de $\Omega^{1}$ sont identifiées à des éléments de $T\left(\Omega^{1}\right) \otimes \mathcal{A}^{o p}$ par tensorisation avec 1 , on note l'application $\int_{\Delta_{k}} \otimes \mathrm{id}:\left(\Omega^{1}\right)^{\otimes k} \otimes \mathcal{A}^{o p} \rightarrow \mathcal{A}^{o p}$ simplement $\int_{\Delta_{k}}$.

On a alors : 
Proposition 2.3. Soit $\mathcal{A}$ une algèbre, limite projective d'algèbres de dimension finie. Soit $\mathbf{a}_{0}, \mathbf{a}_{1}$ des éléments de $\mathcal{A}$ et soit $\boldsymbol{\omega}$ un élément de $\Omega_{\mathbf{a}_{0}, \mathbf{a}_{1}}$. L'holonomie régularisée $\operatorname{Hol}([0,1], \boldsymbol{\omega})$ de l'équation différentielle $d f=\boldsymbol{\omega} f$, définie par (10), est donnée par

$$
\operatorname{Hol}([0,1], \boldsymbol{\omega})=1+\sum_{n \geq 1} I_{[0,1]}^{r e g}(\underbrace{\boldsymbol{\omega}, \ldots, \boldsymbol{\omega}}_{n}) .
$$

Soit $M$ une variété lisse, $U \subset M$ un ouvert, et $\gamma:[0,1] \rightarrow M$ un chemin tel que $\gamma(] 0,1[) \subset U$. Pour $\mathcal{A}, \mathbf{a}_{0}, \mathbf{a}_{1}$ comme ci-dessus, on pose

$$
\Omega_{\mathbf{a}_{0}, \mathbf{a}_{1}}(\gamma):=\left\{\boldsymbol{\omega} \in \Omega^{1}(U, \mathcal{A}) \mid \gamma^{*}(\boldsymbol{\omega}) \in \Omega_{\mathbf{a}_{0}, \mathbf{a}_{1}}\right\} ;
$$

pour $\boldsymbol{\omega}_{1}, \ldots, \boldsymbol{\omega}_{n} \in \Omega_{\mathbf{a}_{0}, \mathbf{a}_{1}}(\gamma)$, on pose $I_{\gamma}^{r e g}\left(\boldsymbol{\omega}_{1}, \ldots, \boldsymbol{\omega}_{n}\right):=I_{[0,1]}^{r e g}\left(\gamma^{*}\left(\boldsymbol{\omega}_{1}\right), \ldots, \gamma^{*}\left(\boldsymbol{\omega}_{n}\right)\right)$. Enfin, pour $\boldsymbol{\omega} \in \Omega_{\mathbf{a}_{0}, \mathbf{a}_{1}}(\gamma)$, on pose $\operatorname{Hol}(\gamma, \boldsymbol{\omega}):=\operatorname{Hol}\left([0,1], \gamma^{*}(\boldsymbol{\omega})\right)$. On a alors

$$
\operatorname{Hol}(\gamma, \boldsymbol{\omega})=1+\sum_{n \geq 1} I_{\gamma}^{r e g}(\underbrace{\boldsymbol{\omega}, \ldots, \boldsymbol{\omega}}_{n}) .
$$

Example 2.4. For $n=2$,

$$
\begin{aligned}
I_{[0,1]}^{r e g}\left(\boldsymbol{\omega}_{1}, \boldsymbol{\omega}_{2}\right)=\int_{\Delta_{2}} & \left(\boldsymbol{\omega}_{1}-\mathbf{a}_{0} d \log (z)\right) \circ\left(\boldsymbol{\omega}_{2}-\mathbf{a}_{1} d \log (1-z)\right)+(-d \log (1-z)) \circ\left(\boldsymbol{\omega}_{2}-\mathbf{a}_{1} d \log (z)\right) \cdot \mathbf{a}_{1} \\
& +\mathbf{a}_{0} \cdot\left(\boldsymbol{\omega}_{1}-\mathbf{a}_{0} d \log (z)\right) \circ(-d \log (z))+\mathbf{a}_{0} \cdot(-d \log (1-z)) \circ(-d \log (z)) \cdot \mathbf{a}_{1} .
\end{aligned}
$$

2.3. Les analogues elliptiques des nombres multizétas. Fixons $\tau \in \mathfrak{H}$. Pour $x \in \mathbb{C}$, on pose

$$
\sigma_{x}^{\tau}(z):=\frac{\theta_{\tau}(z+x)}{\theta_{\tau}(z) \theta_{\tau}(x)} .
$$

Considérant $x$ comme une variable formelle proche de 0 , on voit $\sigma_{x}^{\tau}$ comme un élément de $x^{-1} \operatorname{Mer}(\mathbb{C})[[x]]$, où $\operatorname{Mer}(\mathbb{C})=\{$ fonctions méromorphes définies sur $\mathbb{C}\}$. Plus précisément :

Proposition 2.5. $\sigma_{x}^{\tau}$ admet le développement

$$
\sigma_{x}^{\tau}(z)=\frac{1}{x}+\sum_{n \geq 0} k_{n}^{\tau}(z) x^{n}
$$

avec $k_{0}^{\tau}(z)=\left(\theta_{\tau}^{\prime} / \theta_{\tau}\right)(z)$ et $k_{n}^{\tau}$ finie en 0 et 1 si $n>0$.

Démonstration. Le paramètre $\tau$ étant fixé, on considère $\theta_{\tau}(-)$ comme une fonction de la variable $z$. On a $x \sigma_{x}^{\tau}(z)_{\mid x=0}=1$. De plus,

$$
\left(\sigma_{x}^{\tau}(z)-\frac{1}{x}\right)_{\mid x=0}=\frac{1}{x}\left(\frac{\theta_{\tau}(z+x)}{\theta_{\tau}(z)} \frac{x}{\theta_{\tau}(x)}-1\right)_{\mid x=0}=\frac{\theta_{\tau}^{\prime}}{\theta_{\tau}}(z) .
$$

Enfin, le développement de $\sigma_{x}^{\tau}(z)$ en $z=0$ est, compte tenu de l'imparité de $\theta_{\tau}$

$$
\sigma_{x}^{\tau}(z)=\frac{\theta_{\tau}(x+z)}{\theta_{\tau}(x)} \frac{1}{\theta_{\tau}(z)}=\left(1+z \frac{\theta_{\tau}^{\prime}}{\theta_{\tau}}(x)+O\left(z^{2}\right)\right)\left(\frac{1}{z}+O(z)\right)=\frac{1}{z}+\frac{\theta_{\tau}^{\prime}}{\theta_{\tau}}(x)+O(z) .
$$

Donc

$$
\sigma_{x}^{\tau}(z)-\left(\frac{1}{x}+k_{0}^{\tau}(z)\right)=\left(\frac{\theta_{\tau}^{\prime}}{\theta_{\tau}}(x)-\frac{1}{x}\right)+\left(\frac{1}{z}-\frac{\theta_{\tau}^{\prime}}{\theta_{\tau}}(z)\right)+O(z)
$$

qui est fini en $z=0$. Il s'ensuit que les $k_{n}^{\tau}$ sont finis en 0 . Par symétrie, ils sont également finis en 1 .

Posons $\mathcal{A}:=\mathbb{C}, \mathbf{a}_{0}=\mathbf{a}_{1}:=1$. Alors pour $x \in \mathbb{C}$, la forme $\sigma_{x}^{\tau}(z) d z$ appartient à l'espace $\Omega_{1,1}$ défini par (15).

De même, la forme $e^{2 \pi \mathrm{i} \frac{x z}{\tau}} \sigma_{x}^{\tau}(z) d z$ appartient à l'espace $\Omega_{1,1}(\underline{[0, \tau]})$ défini par (17), où $\underline{[0, \tau]}$ est le chemin linéaire $[0,1] \rightarrow[0, \tau]$ tracé sur $\mathbb{C}$.

On pose alors : 
Définition 2.6. On note $[0,1]$ et $[0, \tau]$ les chemins linéaires $[0,1] \rightarrow[0,1]$ et $[0,1] \rightarrow[0, \tau]$ tracés sur $\mathbb{C}$. Pour $\tau \in \mathfrak{H}$, on pose

$$
\begin{gathered}
I_{x_{1}, \ldots, x_{n}}(\tau):=I_{\underline{[0,1]}}^{r e g}\left(\sigma_{x_{1}}^{\tau} d z, \ldots, \sigma_{x_{n}}^{\tau} d z\right) \\
J_{x_{1}, \ldots, x_{n}}(\tau):=I_{\underline{[0, \tau]}}^{r e g}\left(e^{2 \pi \mathrm{i} \frac{x_{1} z}{\tau}} \sigma_{x_{1}}^{\tau}(z) d z, \ldots, e^{2 \pi \mathrm{i} \frac{x_{n} z}{\tau}} \sigma_{x_{n}}^{\tau}(z) d z\right)
\end{gathered}
$$

ce sont des séries dans $\left(x_{1} \cdots x_{n}\right)^{-1} \mathbb{C}\left[\left[x_{1}, \ldots, x_{n}\right]\right]$.

En utilisant (13), on trouve pour $n \geq 0$

$$
\begin{aligned}
& I_{x_{1}, \ldots, x_{n}}(\tau)=\sum_{\substack{k, l l k, l \geq 0, k+l \leq n-2}} \\
& \int_{\Delta_{n}}\left(\sigma_{x_{k+1}}^{\tau}-d \log (z)\right) \circ\left((-d \log (z))^{\circ a} \amalg(-d \log (1-z))^{\circ b} \amalg\left(\sigma_{x_{k+2}}^{\tau} \circ \cdots \circ \sigma_{x_{n-l-1}}^{\tau}\right)\right) \circ\left(\sigma_{x_{n-l}}^{\tau}-d \log (1-z)\right) \\
& +\int_{\Delta_{n}}(-d \log (1-z)) \circ\left((-d \log (z))^{\circ a} \amalg(-d \log (1-z))^{\circ b} \amalg\left(\sigma_{x_{k+1}}^{\tau} \circ \cdots \circ \sigma_{x_{n-l-2}}^{\tau}\right)\right) \circ\left(\sigma_{x_{n-l-1}}^{\tau}-d \log (1-z)\right) \\
& +\int_{\Delta_{n}}\left(\sigma_{x_{k+2}}^{\tau}-d \log (z)\right) \circ\left((-d \log (z))^{\circ a} \amalg(-d \log (1-z))^{\circ b} \amalg\left(\sigma_{x_{k+3}}^{\tau} \circ \cdots \circ \sigma_{x_{n-l}}^{\tau}\right)\right) \circ(-d \log (z)) \\
& +\int_{\Delta_{n}}(-d \log (1-z)) \circ\left((-d \log (z))^{\circ a} \amalg(-d \log (1-z))^{\circ b} \amalg\left(\sigma_{x_{k+2}}^{\tau} \circ \cdots \circ \sigma_{x_{n-l-1}}^{\tau}\right)\right) \circ(-d \log (z))
\end{aligned}
$$

ce qui donne

$$
I_{x_{1}, \ldots, x_{n}}(\tau)=\int_{\Delta_{n}} \sum_{\substack{k, l, k, l \geq 0, k+l \leq n-2}}(-d \log (z))^{\circ k} \amalg(-d \log (1-z))^{\circ l} \circ\left(\sigma_{x_{k+1}}^{\tau} \circ \cdots \circ \sigma_{x_{n-l}}^{\tau}\right)
$$

(en notant $\sigma_{x}^{\tau}$ à la place de $\left.\sigma_{x}^{\tau}(z) d z\right)$. En particulier, on a

$$
\begin{gathered}
I_{x, y}(\tau)=\int_{\Delta_{2}}\left(\sigma_{x}^{\tau}-d \log (z)\right) \circ\left(\sigma_{y}^{\tau}-d \log (1-z)\right)-d \log (1-z) \circ\left(\sigma_{x}^{\tau}-d \log (1-z)\right) \\
-\left(\sigma_{y}^{\tau}-d \log (z)\right) \circ d \log (z)+d \log (1-z) \circ d \log (z)
\end{gathered}
$$

On a aussi

$$
I_{x}(\tau)=\int_{[0,1]}\left(\sigma_{x}^{\tau}(z) d z-d \log (z)-d \log (1-z)\right)
$$

La fonction $J_{x_{1}, \ldots, x_{n}}(\tau)$ est donnée par les formules analogues, obtenues au moyen des substitutions $[0,1] \rightarrow[0, \tau], \log (z) \rightarrow \log (z / \tau), \log (1-z) \rightarrow \log \left(1-\frac{z}{\tau}\right), \sigma_{x}^{\tau}(z) d z \rightarrow e^{2 \pi \mathrm{i} \frac{x z}{\tau}} \sigma_{x}^{\tau}(z) d z$.

Pour $\underline{d}:=\left(d_{1}, \ldots, d_{n}\right) \in \mathbb{Z}_{\geq-1}^{n}$, on note $I_{\underline{d}}(\tau), J_{\underline{d}}(\tau)$ les nombres complexes définis par

$$
I_{\underline{x}}(\tau)=\sum_{\underline{d} \in \mathbb{Z}_{\geq-1}^{n}} I_{\underline{d}}(\tau) \underline{x} \underline{\underline{d}}, \quad J_{\underline{x}}(\tau)=\sum_{\underline{d} \in \mathbb{Z}_{\geq-1}^{n}} J_{\underline{d}}(\tau) \underline{x}^{\underline{d}},
$$

où $\underline{x}:=\left(x_{1}, \ldots, x_{n}\right)$ et $\underline{x}^{\underline{d}}:=x_{1}^{d_{1}} \cdots x_{n}^{d_{n}}$. On a en particulier $\underbrace{I_{-1, \ldots,-1}}_{n}(\tau)=1 / n !, \underbrace{J_{-1, \ldots,-1}}_{n}(\tau)=$ $\tau^{n} / n !$

On appelle les fonctions $I_{\underline{d}}(\tau), J_{\underline{d}}(\tau)$ les analogues elliptiques des nombres multizétas ; les $I_{x_{1}, \ldots, x_{n}}(\tau), J_{x_{1}, \ldots, x_{n}}(\tau)$ en sont des séries génératrices. Cette terminologie est justifiée par les résultats de la section suivante. 
2.4. Lien avec le développement de $A(\tau), B(\tau)$. Soit

$$
F:=\oplus_{n \geq 0} F_{n}:=\bigoplus_{n \geq 0}\left(x_{1} \cdots x_{n}\right)^{-1} \mathbb{C}\left[\left[x_{1}, \ldots, x_{n}\right]\right] ;
$$

munie du produit $f * g:=h$, avec $h\left(x_{1}, \ldots, x_{n+m}\right):=f\left(x_{1}, \ldots, x_{n}\right) g\left(x_{n+1}, \ldots, x_{n+m}\right)$ pour $f \in F_{n}$, $g \in F_{m}$, c'est une algèbre graduée.

L'algèbre de Lie $\mathfrak{f}_{2}$ est librement engendrée par les éléments $x, y$ et est bigraduée par le degré en ces générateurs. Par élimination de Lazard, la somme directe $\mathfrak{f}_{2} \ominus \mathbb{C} x$ de ses composantes de degré $>0$ en $x$ est l'algèbre de Lie librement engendrée par les $\left[x^{n} y\right]:=(\operatorname{ad} x)^{n}(y), n \geq 0$. Son algèbre enveloppante est donc l'algèbre associative libre sur ces générateurs. On note $\mathcal{A}$ la complétion de cette algèbre enveloppante pour le degré total en $x, y$.

On en déduit :

Lemme 2.7. On a un isomorphisme entre $\mathcal{A}$ et la complétion $\hat{F}:=\prod_{n \geq 0} F_{n}$ de $F$ via

$$
\left[x^{d_{1}} y\right] \cdots\left[x^{d_{n}} y\right] \leftrightarrow x_{1}^{d_{1}-1} \cdots x_{n}^{d_{n}-1} \in F_{n} .
$$

On considère l'algèbre enveloppante de $\mathfrak{f}_{2} \ominus \mathbb{C} x$ comme graduée par le degré total en $x, y$; son algèbre enveloppante est donc graduée avec composantes homogènes de dimension finie, et $\mathcal{A}$ est donc une limite projective d'algèbres de dimension finie.

Posons $\mathbf{a}_{0}=\mathbf{a}_{1}:=t=-[x, y]$ et

$$
\boldsymbol{\omega}(z) d z:=-\frac{\theta_{\tau}(z+\operatorname{ad}(x))}{\theta_{\tau}(z)} \frac{\operatorname{ad}(x)}{\theta_{\tau}(\operatorname{ad}(x))}(y) d z \in \Omega^{1}(] 0,1[, \mathcal{A}) .
$$

Alors $\boldsymbol{\omega}(z) d z \in \Omega_{\mathbf{a}_{0}, \mathbf{a}_{1}}$. On lui associe

$$
I(\tau):=\operatorname{Hol}([0,1], \boldsymbol{\omega}) \in \mathcal{A} .
$$

On pose $\tilde{\boldsymbol{\omega}}(z) d z:=e^{2 \pi \mathrm{i} \frac{z}{\tau}} \boldsymbol{\omega}(z) d z$. Cette forme admet comme pôle $t$ en 0 et $\tau$, donc $\tilde{\boldsymbol{\omega}} \in \Omega_{\mathbf{a}_{0}, \mathbf{a}_{1}}(\underline{[0, \tau]})$, où $[0, \tau]$ est le chemin linéaire $[0,1] \rightarrow[0, \tau]$. On pose alors

$$
J(\tau):=\operatorname{Hol}(\underline{[0, \tau]}, \tilde{\boldsymbol{\omega}}) \in \mathcal{A} .
$$

Sous l'isomorphisme du lemme 2.7, on a

$$
\mathcal{A} \ni \boldsymbol{\omega} \leftrightarrow-\sigma_{x}^{\tau}(z) d z \in F_{1},
$$

d'où

$$
\mathcal{A} \ni I^{r e g}(\underbrace{\boldsymbol{\omega}, \ldots, \boldsymbol{\omega}}_{n}) \leftrightarrow(-1)^{n} I_{[0,1]}^{r e g}\left(\sigma_{x_{n}}^{\tau}, \ldots, \sigma_{x_{1}}^{\tau}\right)=(-1)^{n} I_{x_{n}, \ldots, x_{1}}(\tau) \in F_{n}
$$

d'où

$$
\mathcal{A} \ni I(\tau) \leftrightarrow\left((-1)^{n} I_{x_{n}, \ldots, x_{1}}(\tau)\right)_{n \geq 0} \in \hat{F}
$$

On montre de même la correspondance

$$
\mathcal{A} \ni J(\tau) \leftrightarrow\left((-1)^{n} J_{x_{n}, \ldots, x_{1}}(\tau)\right)_{n \geq 0} \in \hat{F}
$$

Les solutions $f_{0}, f_{1}$ de $d f=\boldsymbol{\omega} f$ (voir section 2.1) sont reliées à $X(z)$ (voir section 1.1) par $X(z)=$ $f_{0}(z)(-2 \pi \mathrm{i})^{t}, X(z-1)=f_{1}(z)(2 \pi \mathrm{i})^{t}$. On en déduit

$$
\operatorname{Ad}\left((2 \pi)^{t}\right)\left(e^{\mathrm{i} \frac{\pi}{2} t} A(\tau) e^{\mathrm{i} \frac{\pi}{2} t}\right)=\operatorname{Hol}([0,1], \Omega) .
$$

On a $B(\tau)=Z(z)^{-1} Z(z+\tau)$, où $Z(z)$ est la solution dans $\mathbf{D}:=\{a+b \tau \mid(a, b) \in] 0,1\left[^{2}\right\}$ de

$$
d Z=\left(\frac{2 \pi \mathrm{i} x}{\tau} d z+\tilde{\boldsymbol{\omega}}(z) d z\right) Z
$$


telle que $Z(z) \sim(-2 \pi \mathrm{i} z)^{t}$ pour $z \rightarrow 0$. Soit $e_{+} \in \operatorname{Der}\left(\mathfrak{f}_{2}\right)$ la dérivation $(x, y) \mapsto(0, x)$. En appliquant l'automorphisme $\exp \left(\frac{2 \pi \mathrm{i}}{\tau} e_{+}\right)$à l'expression reliant $B(\tau)$ avec $Z(z)$, on obtient :

$$
\exp \left(\frac{2 \pi \mathrm{i}}{\tau} e_{+}\right)(B(\tau))=T(z)^{-1} T(z+\tau)
$$

où $T(z)$ est la solution dans $\mathbf{D}$ de $d T=\tilde{\boldsymbol{\omega}}(z) d z \cdot T$, telle que $T(z) \sim(-2 \pi \mathrm{i} z)^{t}$ en $z \rightarrow 0$. Un raisonnement analogue au précédent donne alors

$$
\operatorname{Ad}\left((2 \pi)^{t}\right)\left(e^{-\mathrm{i} \frac{\pi}{2} t} \cdot \exp \left(\frac{2 \pi \mathrm{i}}{\tau} e_{+}\right)(B(\tau)) \cdot e^{-\mathrm{i} \frac{\pi}{2} t}\right)=\operatorname{Hol}([0, \tau], \tilde{\Omega})
$$

(22) et (23) d'une part, (24) et (25) d'autre part impliquent :

Proposition 2.8. Sous l'isomorphisme du Lemme 2.7, on a

$$
\begin{gathered}
\operatorname{Ad}\left((2 \pi)^{t}\right)\left(e^{\mathrm{i} \frac{\pi}{2} t} A(\tau) e^{\mathrm{i} \frac{\pi}{2} t}\right) \leftrightarrow\left(I_{x_{n}, \ldots, x_{1}}(\tau)\right)_{n \geq 0} . \\
\operatorname{Ad}\left((2 \pi)^{t}\right)\left(e^{-\mathrm{i} \frac{\pi}{2} t} \cdot \exp \left(\frac{2 \pi \mathrm{i}}{\tau} e_{+}\right)(B(\tau)) \cdot e^{-\mathrm{i} \frac{\pi}{2} t}\right) \leftrightarrow\left((-1)^{n} J_{x_{n}, \ldots, x_{1}}(\tau)\right)_{n \geq 0} .
\end{gathered}
$$

Remarque 2.9. Le formalisme développé par J. Ecalle utilise une variante de l'isomorphime $F \leftrightarrow \bigoplus_{n \geq 0} \mathbb{C}\left[x_{1}, \ldots, x_{n}\right]$ du lemme 2.7, donné par $\left[x^{d_{1}} y\right] \cdots\left[x^{d_{n}} y\right]=f \leftrightarrow m a_{f}:=x_{1}^{d_{1}} \cdots x_{n}^{d_{n}} \in$ $\mathbb{C}\left[x_{1}, \ldots, x_{n}\right]$.

2.5. Propriétés algébriques et modulaires. Les multizétas elliptiques sont reliés par l'identité modulaire

$$
J_{x_{1}, \ldots, x_{n}}(\tau)=I_{\frac{x_{1}}{\tau}, \ldots, \frac{x_{n}}{\tau}}\left(\frac{-1}{\tau}\right),
$$

qui repose sur l'identité $\sigma_{x}^{-1 / \tau}(z) d z=e^{2 \pi \mathrm{i} x \tau z} \sigma_{\tau x}^{\tau}(\tau z) d(\tau z)$. L'identité (26) traduit l'identité (2) reliant $A(\tau)$ et $B(\tau)$.

Le caractère "de type groupe" de $A(\tau), B(\tau)$ se traduit par les identités

$$
I_{d_{1}, \ldots, d_{n}}(\tau) I_{d_{n+1}, \ldots, d_{n+m}}(\tau)=\sum_{\sigma \in S_{n, m}} I_{d_{\sigma(1)}, \ldots, d_{\sigma(n+m)}}(\tau),
$$

où $S_{n, m}=\left\{\sigma \in S_{n+m} \mid \sigma(i)<\sigma(j)\right.$ si $i<j \leq n$ ou $\left.n+1 \leq i<j\right\}$, ainsi que les identités similaires pour les $J_{\underline{d}}(\tau)$ (qui leur sont équivalentes compte tenu de (26) ).

Les identités (5) se traduisent par

$$
\sum_{k=0}^{n}(-1)^{d_{1}+\cdots+d_{k}} I_{d_{1}, \ldots, d_{k}}(\tau) I_{d_{k+1}, \ldots, d_{n}}(\tau)=0 \quad \text { si } \quad n \geq 1, d_{1}, \ldots, d_{n} \geq-1
$$

et les identités analogues en remplaçant chaque $I_{\underline{d}}$ par $J_{\underline{d}}$ (qui leur sont également équivalentes).

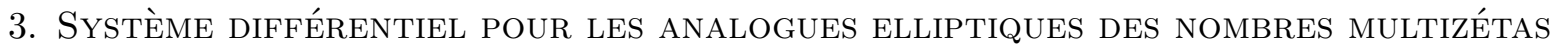

Dans cette section, on montre que les intégrales itérées régularisées définies dans la section 2.2 sont, sous certaines hypothèses, solutions de certains systèmes différentiels (section 3.1). En section 3.2. on applique ce résultat aux analogues elliptiques des nombres multizétas définis en section 2.3.

3.1. Systèmes différentiels pour les intégrales itérées régularisées. Soit $I$ un intervalle de $\mathbb{R}$ contenant 0 . On note $\mathcal{F}$ l'ensemble des 1 -formes lisses sur $I \times] 0,1[$ de la forme $\omega=\omega(t, z) d z$, telles que $\omega-d \log (z)$ admet un prolongement lisse à $I \times[0,1[$ et $\omega-d \log (1-z)$ admet un prolongement lisse à $I \times] 0,1]$. On note $\mathcal{G}$ l'ensemble des 1 -formes lisses sur $I \times[0,1]$ de la forme $g=g(t, z) d t$. On suppose donnés :

- des éléments $\omega_{i}(i=1, \ldots, n)$ et $\psi_{i, i+1}(i=1, \ldots, n-1)$ de $\mathcal{F}$;

- des éléments $g_{i}(i=1, \ldots, n)$ de $\mathcal{G}$, tels que: 
(a) pour $i=1, \ldots, n$, la forme $\omega_{i}(t, z) d z+g_{i}(t, z) d t$ est fermée, ce qui se traduit par l'identité

$$
\frac{\partial \omega_{i}}{\partial t}(t, z)=\frac{\partial g_{i}}{\partial z}(t, z)
$$

sur $I \times] 0,1[$;

(b) pour $i=1, \ldots, n-1$, on a l'identité

$$
g_{i}(t, z) \omega_{i+1}(t, z)-g_{i+1}(t, z) \omega_{i}(t, z)=\left(g_{i}(t, 0)-g_{i+1}(t, 0)\right) \psi_{i, i+1}(t, z)
$$

sur $I \times] 0,1[$;

(c) pour $i=1, \ldots, n$, on a l'identité $g_{i}(t, 0)=g_{i}(t, 1)$ sur $I$.

On se place dans le cadre de la section 2.2 , avec $\mathcal{A}=\mathbb{C}, \mathbf{a}_{0}=\mathbf{a}_{1}=1$, pour définir $\Omega_{1,1}$. Pour tout $\alpha \in \mathcal{F}$ et tout $t \in I$, la restriction $\alpha^{t}$ de $\alpha$ à $\left.\{t\} \times\right] 0,1$ [ appartient à $\Omega_{1,1}$. La définition de $I_{[0,1]}^{\text {reg }}$ en section 2.2 permet alors de définir le nombre $I_{[0,1]}^{r e g}\left(\alpha_{1}^{t}, \ldots, \alpha_{n}^{t}\right)$ pour tous $\alpha_{1}, \ldots, \alpha_{n} \in \mathcal{F}$ et $t \in I$.

Proposition 3.1. Sous les hypothèses du début de la section 3.1 sur $\left(\omega_{i}\right)_{i=1, \ldots, n},\left(\psi_{i, i+1}\right)_{i=1, \ldots, n-1}$, et $\left(g_{i}\right)_{i=1, \ldots, n}$, on a l'identité suivante sur $I$

$$
\begin{aligned}
\frac{d}{d t} I_{[0,1]}^{r e g}\left(\omega_{1}^{t}, \ldots, \omega_{n}^{t}\right)= & -g_{1}^{t}(0) I_{[0,1]}^{r e g}\left(\omega_{2}^{t}, \ldots, \omega_{n}^{t}\right)+g_{n}^{t}(0) I_{[0,1]}^{r e g}\left(\omega_{1}^{t}, \ldots, \omega_{n-1}^{t}\right) \\
& +\sum_{i=1}^{n-1}\left(g_{i}^{t}(0)-g_{i+1}^{t}(0)\right) I_{[0,1]}^{r e g}\left(\omega_{1}^{t}, \ldots, \psi_{i, i+1}^{t}, \ldots, \omega_{n-1}^{t}\right) .
\end{aligned}
$$

La fin de cette section est consacré à la démonstration de cette proposition.

Définition 3.2. On note $\Omega$ l'espace des 1 -formes sur $] 0,1[$ à valeurs dans $\mathbb{C}$, de la forme $\omega(z) d z=$ $\left(\frac{a_{0}}{z}+\frac{a_{1}}{z-1}+f_{0}(z)\right) d z$, où $f_{0}(z)$ est une fonction lisse sur $[0,1]$ et $a_{0}, a_{1} \in \mathbb{C}$. On note $\Omega_{\mathrm{reg} .0}$ et $\Omega_{\mathrm{reg} .1}$ les sous-espaces de $\Omega$ définis par les conditions respectives $a_{0}=0$ et $a_{1}=0$.

On a une application linéaire $\Omega \rightarrow \mathbb{C}^{2}$ donnée par $\omega(z) d z \mapsto\left(a_{0}, a_{1}\right) ; \Omega_{\text {reg.0 }}$ et $\Omega_{\text {reg.1 }}$ sont alors les préimages de $0 \oplus \mathbb{C}$ et $\mathbb{C} \oplus 0$.

Définition 3.3. Pour $m \geq 1$, on définit le sous-espace $\left(\Omega^{\otimes m}\right)_{\text {int }}$ de $\Omega^{\otimes m}$ ainsi:

- si $m=1$, alors $\Omega_{\mathrm{int}}:=\Omega_{\text {reg.0 }} \cap \Omega_{\text {reg. } 1}$;

- si $m \geq 2$, alors $\left(\Omega^{\otimes m}\right)_{\text {int }}:=\Omega_{\text {reg.0 }} \otimes \Omega^{\otimes m-2} \otimes \Omega_{\text {reg.1 }}$.

On identifie $\Omega^{\otimes m}$ à un sous-espace de $\Omega^{m}(] 0,1[m, \mathbb{C})$. Alors l'image de chaque élément de $\left(\Omega^{\otimes m}\right)_{\text {int }}$ est absolument intégrable sur le simplexe $\left.\Delta_{m} \subset\right] 0,1\left[^{m}\right.$. L'intégration sur $\Delta_{m}$ donne alors une application linéaire

$$
\int_{\Delta_{m}}:\left(\Omega^{\otimes m}\right)_{\text {int }} \rightarrow \mathbb{C} .
$$

Définition 3.4. Pour $a \geq 0$, on pose $\left(\Omega^{\otimes a}\right)_{\text {int. } 0}:=\Omega_{\text {reg.0 }} \otimes \Omega^{\otimes a-1}$ si $a>0$, et $\left(\Omega^{\otimes a}\right)_{\text {int. } 0}:=\mathbb{C}$ si $a=0$. Pour $b \geq 0$, on pose $\left(\Omega^{\otimes b}\right)_{\text {int. } 1}:=\Omega^{\otimes a-1} \otimes \Omega_{\text {reg.1 }}$ si $b>0$, et $\left(\Omega^{\otimes b}\right)_{\text {int. } 1}:=\mathbb{C}$ si $b=0$. Enfin, pour $a, b \geq 0$, on pose $\left(\Omega^{\otimes a} \otimes \Omega^{\otimes b}\right)_{\text {int }}:=\left(\Omega^{\otimes a}\right)_{\text {int.0 }} \otimes\left(\Omega^{\otimes b}\right)_{\text {int.1. }}$.

Soit $g$ un élément de $C^{\infty}([0,1], \mathbb{C})$. Alors $d g \in \Omega$. Pour $m$ entier $\geq 1$, on définit l'application linéaire

$$
\operatorname{ins}(d g): \bigoplus_{a+b=m-1} \Omega^{\otimes a} \otimes \Omega^{\otimes b} \rightarrow \Omega^{\otimes m}
$$

comme la somme des applications $\Omega^{\otimes a} \otimes \Omega^{\otimes b} \rightarrow \Omega^{\otimes m}, \alpha \otimes \beta \mapsto \alpha \otimes d g \otimes \beta$ d'insertion de $d g$ dans le produit tensoriel.

Pour $c \geq 0$, on définit une application linéaire

$$
C^{\infty}([0,1], \mathbb{C}) \otimes \Omega^{\otimes c} \rightarrow \Omega^{\otimes c}, \quad f \otimes \omega \mapsto f \cdot \omega
$$


par $f \cdot\left(\gamma_{1} \circ \cdots \circ \gamma_{c}\right):=\left(f \gamma_{1}\right) \circ \cdots \circ \gamma_{c}$ si $c>0$, et $f \cdot 1:=f(1) 1$ pour $c=0(f \gamma$ est le produit de la fonction $f$ et de la 1-forme $\gamma)$. On définit de même une application linéaire

$$
\Omega^{\otimes c} \otimes C^{\infty}([0,1], \mathbb{C}) \rightarrow \Omega^{\otimes c}, \quad \omega \otimes f \mapsto \omega \cdot f
$$

$\operatorname{par}\left(\gamma_{1} \circ \cdots \circ \gamma_{c}\right) \cdot f:=\gamma_{1} \circ \cdots \circ\left(f \gamma_{c}\right)$ si $c>0$, et $1 \cdot f:=f(0) 1$ pour $c=0$.

Pour $g$ dans $C^{\infty}([0,1], \mathbb{C})$, on définit alors des applications linéaires

$$
l(g), r(g): \bigoplus_{a+b=m-1} \Omega^{\otimes a} \otimes \Omega^{\otimes b} \rightarrow \Omega^{\otimes m-1}
$$

comme étant les sommes directes des applications $\Omega^{\otimes a} \otimes \Omega^{\otimes b} \rightarrow \Omega^{\otimes m-1}$ données par $l(g)(\alpha \otimes \beta):=$ $\alpha \otimes(g \cdot \beta), \quad r(g)(\alpha \otimes \beta):=(\alpha \cdot g) \otimes \beta$.

Comme $d g \in \Omega_{\text {reg.0 }} \cap \Omega_{\text {reg.1 }} \subset \Omega$, il existe une application $\left(\bigoplus_{a+b=m-1} \Omega^{\otimes a} \otimes \Omega^{\otimes b}\right)_{\text {int }} \rightarrow\left(\Omega^{\otimes m}\right)_{\text {int }}$, qui sera également notée ins $(d g)$, telle que le diagramme suivant commute

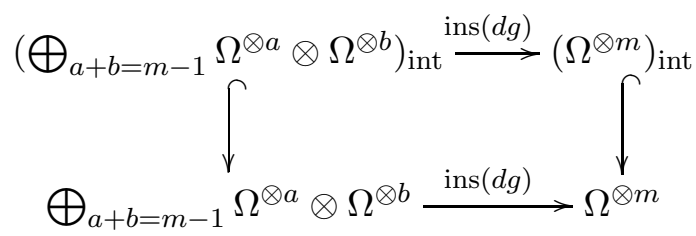

Pour tout $c \geq 0$, les applications $C^{\infty}([0,1], \mathbb{C}) \otimes \Omega^{\otimes c} \rightarrow \Omega^{\otimes c}, f \otimes \omega \mapsto f \cdot \omega$ et $\Omega^{\otimes c} \otimes C^{\infty}([0,1], \mathbb{C}) \rightarrow$ $\Omega^{\otimes c}, \omega \otimes f \mapsto \omega \cdot f$, se restreignent et corestreignent en des applications $C^{\infty}([0,1], \mathbb{C}) \otimes\left(\Omega^{\otimes c}\right)_{\text {int.1 }} \rightarrow$ $\left(\Omega^{\otimes c}\right)_{\text {int.1 }}$ et $\left(\Omega^{\otimes c}\right)_{\text {int. } 0} \otimes C^{\infty}([0,1], \mathbb{C}) \rightarrow\left(\Omega^{\otimes c}\right)_{\text {int.0. }}$. On en déduit l'existence d'applications $l(g), r(g)$ : $\left(\bigoplus_{a+b=m-1} \Omega^{\otimes a} \otimes \Omega^{\otimes b}\right)_{\text {int }} \rightarrow\left(\Omega^{\otimes m-1}\right)_{\text {int }}$, telle que le diagramme

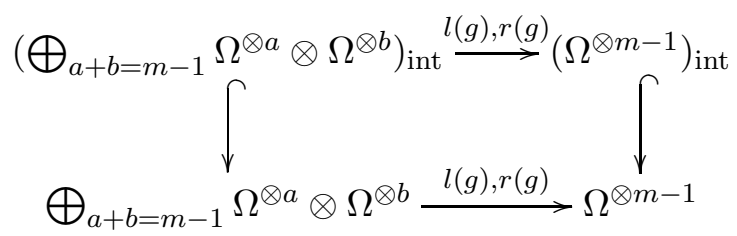

commute. Enfin, le diagramme suivant commute

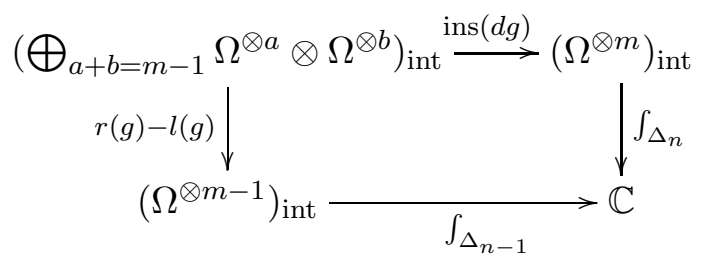

par le théorème de Fubini. On rassemble ces résultats dans le diagramme suivant

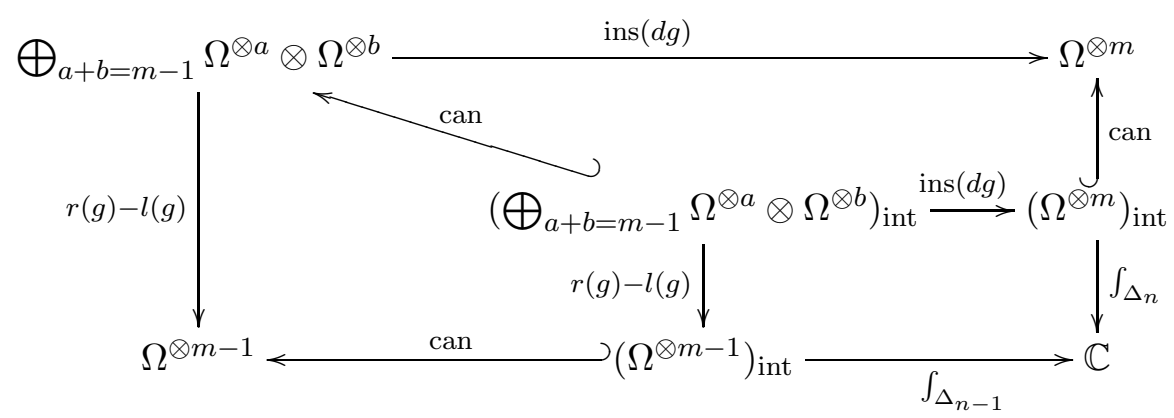

où chacun des carrés commute, et où can sont des inclusions canoniques. 
Posons $T(\Omega):=\oplus_{a \geq 0} \Omega^{\otimes a}$. Les sommes sur $m \geq 0$ des applications linéaires

$$
l(g), r(g): \bigoplus_{a, b \mid a+b=m} \Omega^{\otimes a} \otimes \Omega^{\otimes b} \rightarrow \Omega^{\otimes m}
$$

sont des applications linéaires $l(g), r(g): T(\Omega)^{\otimes 2} \rightarrow T(\Omega)$.

Muni du produit de battage $\amalg$ et du coproduit de déconcaténation $\Delta_{\text {II }}$ donné par $\Delta_{\text {III }}\left(\alpha_{1} \circ\right.$ $\left.\cdots \circ \alpha_{n}\right):=\sum_{k=0}^{n}\left(\alpha_{1} \circ \cdots \circ \alpha_{k}\right) \otimes\left(\alpha_{k+1} \circ \cdots \circ \alpha_{n}\right)$, l'espace $T(\Omega)$ est une bigèbre. Le produit $\amalg$ étant commutatif, les représentations régulières à gauche et à droite de $T(\Omega)$ sont isomorphes ; ceci donne un $T(\Omega)$-module $T(\Omega)$. En utilisant le coproduit, on munit l'espace $T(\Omega)^{\otimes 2}$ d'une structure de $T(\Omega)$-module. On a alors :

Lemme 3.5. L'application linéaire $r(g)-l(g): T(\Omega)^{\otimes 2} \rightarrow T(\Omega)$ est un morphisme de $T(\Omega)$ modules. En d'autres termes, on a

$$
(r(g)-l(g))\left(\left(\alpha^{(1)} \amalg \beta\right) \otimes\left(\alpha^{(2)} \amalg \gamma\right)\right)=\alpha \amalg((r(g)-l(g))(\beta \otimes \gamma))
$$

(égalité dans $T(\Omega)$ ) pour tous $\alpha, \beta, \gamma$ dans $T(\Omega)$, dans laquelle on note $\Delta_{\mathrm{II}}(\alpha)=\alpha^{(1)} \otimes \alpha^{(2)}$.

Démonstration. Notons $a(g):=r(g)-l(g)$. Si $\beta=\gamma=1$, le membre de gauche est égal à $a(g)\left(\alpha^{(1)} \otimes \alpha^{(2)}\right)$ qui est égal, après simplifications, à $\alpha \circ(g \cdot 1)-(1 \cdot g) \circ \alpha=(g(1)-g(0)) \alpha$. D'autre part, le membre de droite est égal à $\alpha \amalg(a(g)(1 \otimes 1))=\alpha \amalg((g \cdot 1)-(1 \cdot g))=(g(1)-g(0)) \alpha$. L'identité (30) est donc satisfaite si $\beta=\gamma=1$.

Si $\beta \in \Omega$ et $\gamma=1$, alors le membre de gauche est égal à $a(g)\left(\left(\alpha^{(1)} \amalg \beta\right) \otimes \alpha^{(2)}\right)$, ce qui d'après l'identité

$$
\forall \alpha \in T(\Omega), \forall \beta \in \Omega, \quad \alpha \amalg \beta=\alpha^{(1)} \circ \beta \circ \alpha^{(2)},
$$

est égal à $a(g)\left(\left(\alpha^{(1)} \circ \beta \circ \alpha^{(2)}\right) \otimes \alpha^{(3)}\right)$. Après simplification, ce dernier terme est égal à $g(1) \alpha^{(1)} \circ$ $\beta \circ \alpha^{(2)}-\alpha^{(1)} \circ(g \beta) \circ \alpha^{(2)}$. D'autre part, le membre de droite est égal à $\alpha \amalg(a(g)(\beta \otimes 1))$. On a $a(g)(\beta \otimes 1)=g(1) \beta-g \beta$, donc compte tenu de (31), le membre de droite est égal à $\alpha^{(1)} \circ(g(1) \beta-$ $g \beta) \circ \alpha^{(2)}$ L'identité (30) est donc satisfaite si $\beta \in \Omega$ et $\gamma=1$. On montre de même que l'identité (30) est satisfaite si $\beta=1$ et $\gamma \in \Omega$.

Si $\beta$ et $\gamma$ appartiennent à $\Omega$, le membre de gauche est égal à $a(g)\left(\left(\alpha^{(1)} \amalg \beta\right) \otimes\left(\alpha^{(2)} \amalg \gamma\right)\right)$, ce qui d'après (31) est égal à $a(g)\left(\left(\alpha^{(1)} \circ \beta \circ \alpha^{(2)}\right) \otimes\left(\alpha^{(3)} \circ \gamma \circ \alpha^{(4)}\right)\right)$, terme qui compte tenu de l'égalité suivante (dans laquelle $T(\Omega)_{+}=\oplus_{a>0} \Omega^{\otimes a}$ )

$$
\forall \alpha, \beta \in T(\Omega), \forall \alpha, \beta \in T(\Omega)_{+}, \quad a(g)\left(\left(\alpha \circ \alpha^{\prime}\right) \otimes\left(\beta^{\prime} \circ \beta\right)\right)=\alpha \circ a(g)\left(\alpha^{\prime} \otimes \beta^{\prime}\right) \circ \beta
$$

est égal à $\alpha^{(1)} \circ a(g)\left(\left(\beta \circ \alpha^{(2)}\right) \otimes\left(\alpha^{(3)} \circ \gamma\right)\right) \circ \alpha^{(4)}$. L'égalité suivante

$$
\forall \beta, \gamma \in \Omega, \forall \alpha \in T(\Omega), \quad a(g)\left(\left(\beta \circ \alpha^{(1)}\right) \otimes\left(\alpha^{(2)} \circ \gamma\right)\right)=\beta \circ \alpha \circ(g \gamma)-(g \beta) \circ \alpha \circ \gamma
$$

implique alors que ce dernier terme est égal à $\alpha^{(1)} \circ\left(\beta \circ \alpha^{(2)} \circ(g \gamma)-(g \beta) \circ \alpha^{(2)} \circ \gamma\right) \circ \alpha^{(3)}$. On a l'identité suivante

$$
\forall \beta, \gamma \in \Omega, \forall \alpha \in T(\Omega), \quad \alpha \amalg(\beta \circ \gamma)=\alpha^{(1)} \circ \beta \circ \alpha^{(2)} \circ \gamma \circ \alpha^{(3)},
$$

qui permet d'exprimer le dernier terme comme $\alpha \varpi(\beta \circ(g \gamma)-(g \beta) \circ \gamma)$, qui est donc $\alpha \amalg a(g)(\beta \otimes \gamma)$, et donc égal au membre de droite. L'identité (30) est donc satisfaite si $\beta=1$ et $\gamma \in \Omega$.

Les deux membres de l'identité (30) sont les valeurs en $\alpha \otimes \beta \otimes \gamma$ de deux applications linéaires $g c h, d t: T(\Omega) \otimes T(\Omega)^{\otimes 2} \rightarrow T(\Omega)$. La décomposition

$$
T(\Omega)^{\otimes 2}=T(\Omega)_{+}^{\otimes 2} \oplus\left(T(\Omega)_{+} \otimes \mathbb{C}\right) \oplus\left(\mathbb{C} \otimes T(\Omega)_{+}\right) \oplus \mathbb{C}^{\otimes 2}
$$

induit par tensorisation avec $T(\Omega)$ une décomposition de la source des applications $g c h, d t$. On a par ailleurs montré l'égalité des restrictions des applications gch et $d t$ aux produits tensoriels de $T(\Omega)$ avec les sous-espaces $\Omega^{\otimes 2} \subset T(\Omega)_{+}^{\otimes 2},(\Omega \otimes \mathbb{C}) \subset(T(\Omega) \otimes \mathbb{C}),(\mathbb{C} \otimes T(\Omega)) \subset(\mathbb{C} \otimes T(\Omega))$, 
et $\mathbb{C}^{\otimes 2} \subset \mathbb{C}^{\otimes 2}$. L'égalité de ces applications linéaires sur les trois premiers espaces est alors une conséquence des identités

$$
\begin{gathered}
\forall \tilde{\beta} \in T(\Omega), \forall \beta_{0} \in T(\Omega)_{+}, \quad a(g)\left(\left(\tilde{\beta} \circ \beta_{0}\right) \otimes 1\right)=\tilde{\beta} \circ a(g)\left(\beta_{0} \otimes 1\right), \\
\forall \tilde{\gamma} \in T(\Omega), \forall \gamma_{0} \in T(\Omega)_{+}, \quad a(g)\left(1 \otimes\left(\gamma_{0} \circ \tilde{\gamma}\right)\right)=a(g)\left(1 \otimes \gamma_{0}\right) \circ \tilde{\gamma}, \\
\forall \tilde{\beta}, \tilde{\gamma} \in T(\Omega), \forall \beta, \gamma \in T(\Omega)_{+}, \quad a(g)\left(\left(\tilde{\beta} \circ \beta_{0}\right) \otimes\left(\gamma_{0} \circ \tilde{\gamma}\right)\right)=\tilde{\beta} \circ a(g)\left(\beta_{0} \otimes \gamma_{0}\right) \circ \tilde{\gamma},
\end{gathered}
$$

et de l'identité

$$
\alpha \amalg(\beta \circ \gamma)=\left(\alpha^{(1)} \amalg \beta\right) \circ\left(\alpha^{(2)} \amalg \gamma\right)
$$

pour $\alpha, \beta, \gamma \in T(\Omega)$.

Montrons par exemple l'identité (30) dans le cas du produit tensoriel de $T(\Omega)$ avec $T(\Omega)_{+}^{\otimes 2}$. Par linéarité, on suppose $\beta \otimes \gamma \in T(\Omega)_{+}^{\otimes 2}$ de la forme $\left(\tilde{\beta} \circ \beta_{0}\right) \otimes\left(\gamma_{0} \circ \tilde{\gamma}\right)$ où $\tilde{\beta}, \tilde{\gamma} \in T(\Omega)$ et $\beta_{0}, \gamma_{0} \in \Omega$. Alors, si $\alpha \in T(\Omega)$,

$$
\begin{aligned}
& a(g)\left(\left(\alpha^{(1)} \amalg \beta\right) \otimes\left(\alpha^{(2)} \amalg \gamma\right)\right)=a(g)\left(\left(\alpha^{(1)} \amalg\left(\tilde{\beta} \circ \beta_{0}\right)\right) \otimes\left(\alpha^{(2)} \amalg\left(\gamma_{0} \circ \tilde{\gamma}\right)\right)\right) \\
& =a(g)\left(\left(\left(\alpha^{(1)} \amalg \tilde{\beta}\right) \circ\left(\alpha^{(2)} \amalg \beta_{0}\right)\right) \otimes\left(\left(\alpha^{(3)} \amalg \gamma_{0}\right) \circ\left(\alpha^{(4)} \amalg \tilde{\gamma}\right)\right)\right) \quad(\text { en utilisant (32) }) \\
& =\left(\alpha^{(1)} \amalg \tilde{\beta}\right) \circ a(g)\left(\left(\alpha^{(2)} \amalg \beta_{0}\right) \otimes\left(\alpha^{(3)} \amalg \gamma_{0}\right)\right) \circ\left(\alpha^{(4)} \amalg \tilde{\gamma}\right) \quad(\text { en utilisant (33) }) \\
& =\left(\alpha^{(1)} \mathrm{\omega} \tilde{\beta}\right) \circ\left(\alpha^{(2)} \amalg a(g)\left(\beta_{0} \otimes \gamma_{0}\right)\right) \circ\left(\alpha^{(3)} \mathrm{\omega} \tilde{\gamma}\right) \quad\left(\text { en utilisant (30) pour } \beta_{0}, \gamma_{0} \in \Omega\right) \\
& =\alpha \amalg\left(\tilde{\beta} \circ a(g)\left(\beta_{0} \otimes \gamma_{0}\right) \circ \tilde{\gamma}\right) \quad(\text { en utilisant (34) }) \\
& =\alpha ш a(g)\left(\left(\tilde{\beta} \circ \beta_{0}\right) \otimes\left(\gamma_{0} \circ \tilde{\gamma}\right)\right) \quad(\text { en utilisant (33) }) \\
& =\alpha ш a(g)(\beta \otimes \gamma) .
\end{aligned}
$$

Compte tenu de ce que $\left(T(\Omega), \amalg, \Delta_{\text {II }}\right)$ est une bigèbre, (30) implique :

$\forall \alpha, \beta, \gamma, \delta \in T(\Omega), \quad(r(g)-l(g))\left(\left(\alpha^{(1)} \amalg \beta \amalg \delta^{(1)}\right) \otimes\left(\alpha^{(2)} \amalg \gamma \amalg \delta^{(2)}\right)\right)=\alpha \amalg((r(g)-l(g))(\beta \otimes \gamma)) \amalg \delta$

(égalité dans $T(\Omega)$ ).

Pour $\breve{\omega}_{1}, \ldots, \breve{\omega}_{n} \in \Omega_{1,1}$, on pose

$$
\operatorname{int}\left(\breve{\omega}_{1}, \ldots, \breve{\omega}_{n}\right):=\sum_{a, b \mid a+b \leq n}(-1)^{a+b}(d \ln (z))^{\circ a} \amalg\left(\breve{\omega}_{a+1} \circ \cdots \circ \breve{\omega}_{n-b}\right) \amalg(d \ln (1-z))^{\circ b} \in \Omega^{\otimes n} .
$$

On a vu en section 2.2 que $\operatorname{int}\left(\breve{\omega}_{1}, \ldots, \breve{\omega}_{n}\right)=\sum_{\substack{\epsilon, \eta \in\{0,1\} \\ k, l \mid k+l \leq n-2}} \operatorname{int}_{k, l}^{\epsilon, \eta}\left(\breve{\omega}_{1}, \ldots, \breve{\omega}_{n}\right)$, où

$$
\begin{aligned}
& \operatorname{int}_{k, l}^{0,0}\left(\breve{\omega}_{1}, \ldots, \breve{\omega}_{n}\right) \\
& =\left(\breve{\omega}_{k+1}-d \log (z)\right) \circ\left((-d \log (z))^{\circ k} \amalg(-d \log (1-z))^{\circ l} \amalg\left(\breve{\omega}_{k+2} \circ \cdots \circ \breve{\omega}_{n-l-1}\right)\right) \circ\left(\breve{\omega}_{n-l}-d \log (1-z)\right), \\
& \quad \operatorname{int}_{k, l}^{1,0}\left(\breve{\omega}_{1}, \ldots, \breve{\omega}_{n}\right) \\
& \quad=\left(\breve{\omega}_{k+2}-d \log (z)\right) \circ\left((-d \log (z))^{\circ k} \amalg(-d \log (1-z))^{\circ l} \amalg\left(\breve{\omega}_{k+3} \circ \cdots \circ \breve{\omega}_{n-l}\right)\right) \circ(-d \log (z)), \\
& \operatorname{int}_{k, l}^{0,1}\left(\breve{\omega}_{1}, \ldots, \breve{\omega}_{n}\right) \\
& =(-d \log (1-z)) \circ\left((-d \log (z))^{\circ k} \amalg(-d \log (1-z))^{\circ l} \amalg\left(\breve{\omega}_{k+1} \circ \cdots \circ \breve{\omega}_{n-l-2}\right)\right) \circ\left(\breve{\omega}_{n-l-1}-d \log (1-z)\right),
\end{aligned}
$$




$$
\begin{aligned}
& \operatorname{int}_{k, l}^{0,0}\left(\breve{\omega}_{1}, \ldots, \breve{\omega}_{n}\right) \\
& =(-d \log (1-z)) \circ\left((-d \log (z))^{\circ k} \amalg(-d \log (1-z))^{\circ l} \amalg\left(\breve{\omega}_{k+2} \circ \cdots \circ \breve{\omega}_{n-l-1}\right)\right) \circ(-d \log (z)) .
\end{aligned}
$$

Chaque $\operatorname{int}_{k, l}^{\epsilon, \eta}\left(\breve{\omega}_{1}, \ldots, \breve{\omega}_{n}\right)$ appartient à $\left(\Omega^{\otimes n}\right)_{\text {int }}$, donc $\operatorname{int}\left(\breve{\omega}_{1}, \ldots, \breve{\omega}_{n}\right)$ aussi. On a aussi posé

$$
I_{[0,1]}^{r e g}\left(\breve{\omega}_{1}, \ldots, \breve{\omega}_{n}\right)=\int_{\Delta_{n}} \operatorname{int}\left(\breve{\omega}_{1}, \ldots, \breve{\omega}_{n}\right) .
$$

Soit $\breve{g}_{1}, \ldots, \breve{g}_{n} \in C^{\infty}([0,1], \mathbb{C})$ tels que $\breve{g}_{i}(0)=\breve{g}_{i}(1)$; pour $n \in\{1, \ldots, n\}$, on définit l'élément suivant $\Omega^{\otimes n}$

$$
\delta_{c} \operatorname{int}\left(\breve{\omega}_{1}, \ldots, \breve{\omega}_{n}\right):=\sum_{\substack{a, b \mid a+b \leq n \\ a+1 \leq c \leq n-b}}(-1)^{a+b}(-1)^{a+b}(d \ln (z))^{\circ a} \amalg\left(\breve{\omega}_{a+1} \circ \cdots \circ d \breve{g}_{c} \circ \cdots \circ \breve{\omega}_{n-b}\right) \amalg(d \ln (1-z))^{\circ b} .
$$

On définit $\delta_{c} \operatorname{int}_{k, l}^{\epsilon, \eta}\left(\breve{\omega}_{1}, \ldots, \breve{\omega}_{n}\right)$ comme étant 0 si $c \notin\{k+1, \ldots, n-l\}$, et comme étant le résultat du remplacement du terme comprenant $\breve{\omega}_{c}$ (qui peut être $\breve{\omega}_{c}, \breve{\omega}_{c}-d \log (z)$ ou $\breve{\omega}_{c}-d \log (1-z)$ ) par $d \breve{g}_{c}$. Par exemple, on a

$$
\begin{aligned}
& \delta_{c} \operatorname{int}_{k, l}^{0,0}\left(\breve{\omega}_{1}, \ldots, \breve{\omega}_{n}\right):= \\
& \delta_{k+1, c} d \breve{g}_{c} \circ\left((-d \log (z))^{\circ k} \amalg(-d \log (1-z))^{\circ l} \amalg\left(\breve{\omega}_{k+2} \circ \cdots \circ \breve{\omega}_{n-l-1}\right)\right) \circ\left(\breve{\omega}_{n-l}-d \log (1-z)\right) \\
& +\delta_{k+2 \leq c \leq n-l-1}\left(\breve{\omega}_{k+1}-d \log (z)\right) \circ \\
& \circ\left((-d \log (z))^{\circ k} \amalg(-d \log (1-z))^{\circ l} \amalg\left(\breve{\omega}_{k+2} \circ \cdots \circ d \breve{g}_{c} \circ \cdots \circ \breve{\omega}_{n-l-1}\right)\right) \circ\left(\breve{\omega}_{n-l}-d \log (1-z)\right) \\
& +\delta_{n-l, c}\left(\breve{\omega}_{k+1}-d \log (z)\right) \circ\left((-d \log (z))^{\circ k} \amalg(-d \log (1-z))^{\circ l} \amalg\left(\breve{\omega}_{k+2} \circ \cdots \circ \breve{\omega}_{n-l-1}\right)\right) \circ d \breve{g}_{c}
\end{aligned}
$$

(où $\delta_{k+2 \leq c \leq n-l-1}=1$ si $k+2 \leq c \leq n-l-1$, et $=0$ sinon).

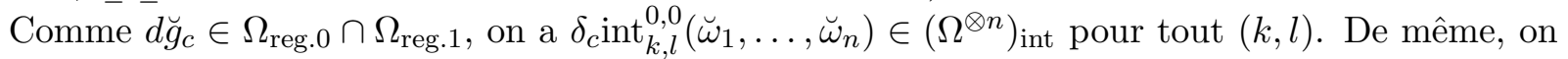
a pour $(\epsilon, \eta) \in\{(1,0),(0,1),(1,1)\}$ et tout $(k, l), \delta_{c}$ int $_{k, l}^{\epsilon, \eta}\left(\breve{\omega}_{1}, \ldots, \breve{\omega}_{n}\right) \in\left(\Omega^{\otimes n}\right)_{\text {int }}$. On en déduit

$$
\delta_{c} \operatorname{int}\left(\breve{\omega}_{1}, \ldots, \breve{\omega}_{n}\right) \in\left(\Omega^{\otimes n}\right)_{\text {int }} .
$$

On définit l'élément $\operatorname{rel}_{c} \in \bigoplus_{a, b \mid a+b=n-1} \Omega^{\otimes a} \otimes \Omega^{\otimes b}$ par

$$
\begin{array}{r}
\operatorname{rel}_{c}:=\sum_{\substack{a, b \mid a+b \leq n \\
a+1 \leq c \leq n-b}} \sum_{\substack{a=a^{\prime}+a^{\prime \prime} \\
b=b^{\prime}+b^{\prime \prime}}}(-1)^{a+b}\left((d \ln (z))^{\circ a^{\prime}} \amalg\left(\breve{\omega}_{a+1} \circ \cdots \circ \breve{\omega}_{c-1}\right) \amalg(d \ln (1-z))^{\circ b^{\prime}}\right) \otimes \\
\otimes\left((d \ln (z))^{\circ a^{\prime \prime}} \amalg\left(\breve{\omega}_{c+1} \circ \cdots \circ \breve{\omega}_{n-b}\right) \amalg(d \ln (1-z))^{\circ b^{\prime \prime}}\right)
\end{array}
$$

On définit $\left(\operatorname{rel}_{c}\right)_{k, l}^{0,0} \in \bigoplus_{a, b \mid a+b=n-1}\left(\Omega^{\otimes a} \otimes \Omega^{\otimes b}\right)_{\text {int }}$ par

$$
\begin{aligned}
& \left(\operatorname{rel}_{c}\right)_{k, l}^{0,0}:=\delta_{k+1, c} \cdot 1 \otimes\left(\left((-d \log (z))^{\circ k} \amalg(-d \log (1-z))^{\circ l} \amalg\left(\breve{\omega}_{k+2} \circ \cdots \circ \breve{\omega}_{n-l-1}\right)\right) \circ\left(\breve{\omega}_{n-l}-d \log (1-z)\right)\right) \\
& +\delta_{k+2 \leq c \leq n-l-1}\left(\breve{\omega}_{k+1}-d \log (z)\right) \circ \\
& \circ\left((-d \log (z))^{\circ k} \amalg(-d \log (1-z))^{\circ l} \amalg\left(\breve{\omega}_{k+2} \circ \cdots \circ d \breve{g}_{c} \circ \cdots \circ \breve{\omega}_{n-l-1}\right)\right) \circ\left(\breve{\omega}_{n-l}-d \log (1-z)\right) \\
& +\sum_{\substack{k^{\prime}, k^{\prime \prime}\left|k=k^{\prime}+k^{\prime \prime} \\
l^{\prime}, l^{\prime \prime}\right| l=l^{\prime}+l^{\prime \prime}}} \delta_{k+2 \leq c \leq n-l-1}\left(\left(\breve{\omega}_{k+1}-d \log (z)\right) \circ\left((-d \ln (z))^{\circ k^{\prime}} \amalg(-d \ln (1-z))^{\circ l^{\prime}} \amalg\left(\breve{\omega}_{k+2} \circ \cdots \circ \breve{\omega}_{c-1}\right)\right)\right) \otimes \\
& \otimes\left(\left(\left(\breve{\omega}_{c+1} \circ \cdots \circ \breve{\omega}_{n-l-1}\right) \amalg(-d \ln (z))^{\circ k^{\prime \prime}} \amalg(-d \ln (1-z))^{\circ l^{\prime \prime}}\right) \circ\left(\breve{\omega}_{n-l}-d \log (1-z)\right)\right) \\
& +\delta_{n-l, c}\left(\left(\breve{\omega}_{k+1}-d \log (z)\right) \circ\left((-d \log (z))^{\circ k} \amalg(-d \log (1-z))^{\circ l} \amalg\left(\breve{\omega}_{k+2} \circ \cdots \circ \breve{\omega}_{n-l-1}\right)\right)\right) \otimes 1
\end{aligned}
$$


et on définit de façon analogue les autres $\left(\operatorname{rel}_{c}\right)_{k, l}^{\epsilon, \eta}$ dans $\bigoplus_{a, b \mid a+b=n-1}\left(\Omega^{\otimes a} \otimes \Omega^{\otimes b}\right)_{\text {int }}$. Alors $\operatorname{rel}_{c}=$ $\sum_{\epsilon, \eta \in\{0,1\}} \sum_{k, l \mid k+l \leq n-2}\left(\mathrm{rel}_{c}\right)_{k, l}^{\epsilon, \eta}$, donc

$$
\operatorname{rel}_{c} \in \bigoplus_{a, b \mid a+b=n-1}\left(\Omega^{\otimes a} \otimes \Omega^{\otimes b}\right)_{\mathrm{int}} .
$$

Compte tenu de (35), on a

$$
\begin{aligned}
\left(r\left(\breve{g}_{c}\right)-l\left(\breve{g}_{c}\right)\right)\left(\operatorname{rel}_{c}\right)= & \sum_{\substack{a, b \mid a+b \leq n \\
a+1 \leq c \leq n-b}}(-1)^{a+b}(d \log (z))^{\circ a} \amalg(d \log (1-z))^{\circ b} \amalg \\
& \amalg\left(r\left(\breve{g}_{c}\right)-l\left(\breve{g}_{c}\right)\right)\left(\left(\breve{\omega}_{a+1} \circ \cdots \circ \breve{\omega}_{c-1}\right) \otimes\left(\breve{\omega}_{c+1} \circ \cdots \circ \breve{\omega}_{n-b}\right)\right)
\end{aligned}
$$

On a donc

$$
\begin{aligned}
& \sum_{c=1}^{n}\left(l\left(\breve{g}_{c}\right)-r\left(\breve{g}_{c}\right)\right)\left(\operatorname{rel}_{c}\right)=\sum_{a, b \mid a+b \leq n}(-1)^{a+b}(d \log (z))^{\circ a} \amalg(d \log (1-z))^{\circ b} \amalg \\
& \amalg\left(-\breve{g}_{a+1}(0)\left(\breve{\omega}_{a+2} \circ \cdots \circ \breve{\omega}_{n-b}\right)+\left(\breve{\omega}_{a+1} \circ \cdots \circ \breve{\omega}_{n-b-1}\right) g_{n-b}(0)\right. \\
& \left.+\sum_{i=a+1}^{n-b-1} \breve{\omega}_{a+1} \circ \cdots \circ\left(\breve{g}_{i} \breve{\omega}_{i+1}-\breve{g}_{i+1} \breve{\omega}_{i}\right) \circ \cdots \circ \breve{\omega}_{n-b}\right) .
\end{aligned}
$$

Supposons que pour $i=1, \ldots, n-1$, on dispose de $\breve{\psi}_{i, i+1} \in \Omega_{1,1}$ tel que $\breve{g}_{i} \breve{\omega}_{i+1}-\breve{g}_{i+1} \breve{\omega}_{i}=$ $\left(\breve{g}_{i}(0)-\breve{g}_{i+1}(0)\right) \breve{\psi}_{i, i+1}$. Alors

$$
\begin{aligned}
& \sum_{c=1}^{n}\left(l\left(\breve{g}_{c}\right)-r\left(\breve{g}_{c}\right)\right)\left(\operatorname{rel}_{c}\right)=\sum_{a, b \mid a+b \leq n}(-1)^{a+b}(d \log (z))^{\circ a} \amalg(d \log (1-z))^{\circ b} \amalg \\
& \amalg\left(-\breve{g}_{a+1}(0)\left(\breve{\omega}_{a+2} \circ \cdots \circ \breve{\omega}_{n-b}\right)+\left(\breve{\omega}_{a+1} \circ \cdots \circ \breve{\omega}_{n-b-1}\right) \breve{g}_{n-b}(0)\right. \\
& \left.+\sum_{i=a+1}^{n-b-1}\left(\breve{g}_{i}(0)-\breve{g}_{i+1}(0)\right) \breve{\omega}_{a+1} \circ \cdots \circ \breve{\psi}_{i, i+1} \circ \cdots \circ \breve{\omega}_{n-b}\right)
\end{aligned}
$$


(égalité dans $\Omega^{\otimes n-1}$ ) D'autre part, en décomposant le développement de $\operatorname{int}\left(\breve{\omega}_{1}, \ldots, \breve{\psi}_{i, i+1}, \ldots, \breve{\omega}_{n}\right)$ selon les valeurs de $a, b$, on a le développement suivant dans $\Omega^{\otimes n-1}$

$$
\begin{aligned}
& -\breve{g}_{1}(0) \operatorname{int}\left(\breve{\omega}_{2}, \ldots, \breve{\omega}_{n}\right)+\breve{g}_{n}(0) \operatorname{int}\left(\breve{\omega}_{1}, \ldots, \breve{\omega}_{n-1}\right)+\sum_{i=1}^{n-1}\left(\breve{g}_{i}(0)-\breve{g}_{i+1}(0)\right) \operatorname{int}\left(\breve{\omega}_{1}, \ldots, \breve{\psi}_{i, i+1}, \ldots, \breve{\omega}_{n}\right) \\
& =-\breve{g}_{1}(0) \sum_{a, b \mid a+b \leq n-1}(-d \ln (z))^{\circ a} \amalg(-d \ln (1-z))^{\circ b} \amalg\left(\breve{\omega}_{a+2} \circ \cdots \circ \breve{\omega}_{n-b}\right) \\
& +\breve{g}_{n}(0) \sum_{a, b \mid a+b \leq n-1}(-d \ln (z))^{\circ a} \mathrm{\omega}(-d \ln (1-z))^{\circ b} \amalg\left(\breve{\omega}_{a+1} \circ \cdots \circ \breve{\omega}_{n-b-1}\right) \\
& +\sum_{i=1}^{n-1}\left(\breve{g}_{i}(0)-\breve{g}_{i+1}(0)\right) \sum_{\substack{a, b \mid a+b \leq n-1 \\
a \leq i-1, b \leq n-i-1}}(-d \ln (z))^{\circ a} \amalg(-d \ln (1-z))^{\circ b} \amalg\left(\breve{\omega}_{a+1} \circ \cdots \circ \breve{\psi}_{i, i+1} \circ \cdots \circ \breve{\omega}_{n-b}\right) \\
& +\sum_{i=1}^{n-1}\left(\breve{g}_{i}(0)-\breve{g}_{i+1}(0)\right) \sum_{\substack{a, b \mid a+b \leq n-1 \\
a \geq i-1}}(-d \ln (z))^{\circ a} \amalg(-d \ln (1-z))^{\circ b} \amalg\left(\breve{\omega}_{a+2} \circ \cdots \circ \breve{\omega}_{n-b}\right) \\
& +\sum_{i=1}^{n-1}\left(\breve{g}_{i}(0)-\breve{g}_{i+1}(0)\right) \sum_{\substack{a, b \mid a+b \leq n-1 \\
b \geq n-i}}(-d \ln (z))^{\circ a} \omega(-d \ln (1-z))^{\circ b} \amalg\left(\breve{\omega}_{a+1} \circ \cdots \circ \breve{\omega}_{n-b-1}\right)
\end{aligned}
$$

Après inversion des signes somme dans le quatrième terme du membre de droite de (37), on voit que la somme de ce terme et du premier terme de ce membre de droite est égale au premier terme du membre de droite de (36). De même, la somme des deuxième et cinquième termes du membre de droite de (37) est égale au deuxième terme du membre de droite de (36). Enfin, les troisèmes termes des membres de droite de (36) et (37) sont égaux. On a donc l'égalité suivante

$$
\begin{aligned}
& \sum_{c=1}^{n}\left(l\left(\breve{g}_{c}\right)-r\left(\breve{g}_{c}\right)\right)\left(\operatorname{rel}_{c}\right) \\
& =-\breve{g}_{1}(0) \operatorname{int}\left(\breve{\omega}_{2}, \ldots, \breve{\omega}_{n}\right)+\breve{g}_{n}(0) \operatorname{int}\left(\breve{\omega}_{1}, \ldots, \breve{\omega}_{n-1}\right)+\sum_{i=1}^{n-1}\left(\breve{g}_{i}(0)-\breve{g}_{i+1}(0)\right) \operatorname{int}\left(\breve{\omega}_{1}, \ldots, \breve{\psi}_{i, i+1}, \ldots, \breve{\omega}_{n}\right)
\end{aligned}
$$

dans $\Omega^{\otimes n-1}$, et donc dans $\left(\Omega^{\otimes n-1}\right)_{\text {int }}$.

Rappelons que pour $c \in\{1, \ldots, n\}, \operatorname{rel}_{c} \in\left(\bigoplus_{a+b=n-1} \Omega^{\otimes a} \otimes \Omega^{\otimes b}\right)$ int est tel que ins $\left(d \breve{g}_{c}\right)\left(\operatorname{rel}_{c}\right)=$ $\delta_{c} \operatorname{int}\left(\breve{\omega}_{1}, \ldots, \breve{\omega}_{n}\right)$. On en déduit (voir (29) $)$ que $\left(r\left(\breve{g}_{c}\right)-l\left(\breve{g}_{c}\right)\right)\left(\operatorname{rel}_{c}\right) \in\left(\Omega^{\otimes n-1}\right)_{\text {int }}$ et $\int_{\Delta_{n}} \delta_{c} \operatorname{int}\left(\breve{\omega}_{1}, \ldots, \breve{\omega}_{n}\right)=$ $\int_{\Delta_{n-1}}\left(r\left(\breve{g}_{c}\right)-l\left(\breve{g}_{c}\right)\right)\left(\operatorname{rel}_{c}\right)$. En sommant sur $c=1, \ldots, n$, et en utilisant (38), on en déduit

$$
\int_{\Delta_{n}} \delta_{c} \operatorname{int}\left(\breve{\omega}_{1}, \ldots, \breve{\omega}_{n}\right)=\int_{\Delta_{n-1}}(\text { membre de droite de (38) }) .
$$

On se place maintenant dans le cadre de l'énoncé de la proposition 3.1. Fixons $t \in I$ et posons $\breve{\omega}_{i}:=\omega_{i}^{t}, \breve{g}_{i}:=g_{i}^{t}, \breve{\psi}_{i, i+1}:=\psi_{i, i+1}^{t}$. Alors les hypothèses sur les $\breve{\omega}_{i}, \breve{g}_{i}, \breve{\psi}_{i, i+1}$ sont satisfaites, et $\delta_{c} \operatorname{int}\left(\breve{\omega}_{1}, \ldots, \breve{\omega}_{n}\right)=(d / d t) \operatorname{int}\left(\omega_{1}^{t}, \ldots, \omega_{n}^{t}\right)$, donc le membre de gauche de (39) s'identifie au membre de gauche de (28). De même, le membre de droite de (39) s'identifie au membre de droite de (28). Ceci montre (28) et termine la démonstration de la proposition 3.1 .

3.2. Systèmes différentiels pour les analogues elliptiques des nombres multizétas. Soient $x_{1}, \ldots, x_{n}$ des nombres complexes (ou des variables formelles proches de 0 ). 
Définition 3.6. Pour $i \in\{1, \ldots, n\}$ et $(\tau, z) \in \mathfrak{H} \times] 0,1[$, on pose

$$
\omega_{i}(\tau, z) d z:=\sigma_{x_{i}}^{\tau}(z) d z, \quad g_{i}(\tau, z) d \tau:=\frac{\partial}{\partial x_{i}}\left(\frac{1}{2 \pi \mathrm{i}} \sigma_{x_{i}}^{\tau}(z)\right) d \tau
$$

et pour $i \in\{1, \ldots, n-1\}$ et $(\tau, z) \in \mathfrak{H} \times] 0,1[$,

$$
\psi_{i, i+1}(\tau, z) d z:=\sigma_{x_{i}+x_{i+1}}^{\tau}(z) d z
$$

On a vu que pour chaque couple $(\tau, x), \sigma_{x}^{\tau}(z) d z$ est dans $\Omega_{1,1}$, ce qui implique que les $\omega_{i}(i=$ $1, \ldots, n)$ et les $\psi_{i, i+1}(i=1, \ldots, n-1)$ sont dans $\mathcal{F}$. De plus, on a le développement

$$
\begin{aligned}
& \frac{1}{2 \pi \mathrm{i}} \frac{1}{\theta_{\tau}(z)} \frac{\theta_{\tau}(z+x)}{\theta_{\tau}(x)}=\frac{1}{2 \pi \mathrm{i}}\left(\frac{1}{z}+O(z)\right) \frac{\theta_{\tau}(z+x)}{\theta_{\tau}(x)} \\
& =\frac{1}{2 \pi \mathrm{i} \theta_{\tau}(x)}\left(\frac{\theta_{\tau}(x)}{z}+\theta_{\tau}^{\prime}(x)+O(z)\right)=\frac{1}{2 \pi \mathrm{i}}\left(\frac{1}{z}+\frac{\theta_{\tau}^{\prime}}{\theta_{\tau}}(x)+O(z)\right)
\end{aligned}
$$

d'où le fait que à $(\tau, x)$ fixé, $z \mapsto g_{x}(\tau, z)$ est lisse en 0 , avec

$$
g_{x}(\tau, z)=\frac{\partial}{\partial x}\left(\frac{1}{2 \pi \mathrm{i}} \frac{\theta_{\tau}}{\theta_{\tau}}(x)\right)+O(z)
$$

d'où l'on déduit

$$
g_{x}(\tau, 0)=\frac{\partial}{\partial x}\left(\frac{1}{2 \pi \mathrm{i}} \frac{\theta_{\tau}}{\theta_{\tau}}(x)\right) .
$$

L'invariance de $z \mapsto \theta_{\tau}(z)$ sous $z \mapsto 1-z$ implique que $g_{z}(\tau, z)$ est également lisse en 1 , avec $g_{x}(\tau, 0)=g_{x}(\tau, 1)$.

Dans [CEE], trois lignes après l'équation (14), on montre l'égalité

$$
\partial_{\tau}\left(\frac{\theta_{\tau}(z+x)}{\theta_{\tau}(z) \theta_{\tau}(x)}\right)=\frac{1}{2 \pi \mathrm{i}} \partial_{z} \partial_{x}\left(\frac{\theta_{\tau}(z+x)}{\theta_{\tau}(z) \theta_{\tau}(x)}\right)
$$

qui implique immédiatement

pour $i \in\{1, \ldots, n\}$.

$$
\frac{\partial \omega_{i}}{\partial \tau}(\tau, z)=\frac{\partial g_{i}}{\partial z}(\tau, z)
$$

La fonction de Weierstrass est définie par $\wp_{\tau}(z)=\sum_{a \in \mathbb{Z}+\tau \mathbb{Z}}^{\prime}\left((z+a)^{-2}-a^{-2}\right)$, où $\sum^{\prime}$ signifie que le terme $a^{-2}$ n'est pas pris en compte lorsque $a=0$. On pose alors

$$
\tilde{\wp}_{\tau}(z):=\wp_{\tau}(z)+G_{2}(\tau)=\sum_{m \in \mathbb{Z}}\left(\sum_{n}^{\prime}(z+n+m \tau)^{-2}\right) .
$$

Lemme 3.7. On a les développements de Laurent suivants en $x=0$

$$
\frac{\theta_{\tau}^{\prime}}{\theta_{\tau}}(x)=\frac{1}{x}-G_{2}(\tau) x-G_{4}(\tau) x^{3}-\cdots, \quad \wp_{\tau}(x)=\frac{1}{x^{2}}+3 G_{4}(\tau) x^{2}+5 G_{6}(\tau) x^{4}+\cdots .
$$

Démonstration. Le deuxième développement provient de $(x+a)^{-2}=a^{-2}-2 x a^{-3}+\cdots$.

D'après [Po, Thm. 3.9, $\wp_{\tau}=-\left(\sigma^{\prime} / \sigma\right)^{\prime}$, où on pose $\sigma(z):=e^{\frac{1}{2} G_{2}(\tau) z^{2}} \theta_{\tau}(z)$. Donc $\left(\theta_{\tau}^{\prime} / \theta_{\tau}\right)^{\prime}(z)=$ $-\wp_{\tau}(z)-G_{2}(\tau)$, ce qui détermine le développement de $\theta_{\tau}^{\prime} / \theta_{\tau}$ à une constante additive près. Cette constante est déterminée par le fait que $\theta_{\tau}^{\prime} / \theta_{\tau}$ est une fonction impaire.

On en déduit

$$
\tilde{\wp}_{\tau}(x)=\sum_{n \geq-1}(2 n+1) G_{2 n+2}(\tau) x^{2 n}=-\left(\frac{\theta_{\tau}^{\prime}}{\theta_{\tau}}\right)^{\prime}(x),
$$

où on a posé $G_{0}(\tau):=-1$. L'équation (40) implique alors

$$
g_{i}(0, \tau)=g_{i}(1, \tau)=-\frac{1}{2 \pi \mathrm{i}} \tilde{\wp}_{\tau}\left(x_{i}\right) .
$$


Lemme 3.8. On a l'identité

$$
\forall x, y \in \mathbb{C}, \quad\left(\partial_{x} \sigma_{x}^{\tau}\right) \sigma_{y}^{\tau}-\sigma_{x}^{\tau}\left(\partial_{y} \sigma_{y}^{\tau}\right)=\sigma_{x+y}^{\tau}\left(\wp_{\tau}(y)-\wp_{\tau}(x)\right),
$$

Démonstration. Le membre de gauche a le même comportement que le membre de droite sous les transformations de la variable muette $z \mapsto z+1, z \mapsto z+\tau$; pour étudier son comportement en $z=0$, on le transforme ainsi

$\left(\partial_{x} \sigma_{x}^{\tau}\right) \sigma_{y}^{\tau}(z)-\sigma_{x}^{\tau}\left(\partial_{y} \sigma_{y}^{\tau}\right)(z)=\sigma_{x}^{\tau} \sigma_{y}^{\tau}\left(\frac{\partial_{x} \sigma_{x}^{\tau}}{\sigma_{x}^{\tau}}-\frac{\partial_{y} \sigma_{y}^{\tau}}{\sigma_{y}^{\tau}}\right)(z)=\sigma_{x}^{\tau} \sigma_{y}^{\tau}\left(\frac{\theta_{\tau}^{\prime}}{\theta_{\tau}}(z+x)-\frac{\theta_{\tau}^{\prime}}{\theta_{\tau}}(x)-\frac{\theta_{\tau}^{\prime}}{\theta_{\tau}}(z+y)+\frac{\theta_{\tau}^{\prime}}{\theta_{\tau}}(y)\right)$

dont le développement est $\frac{1}{z^{2}} \times z \times\left(\left(\frac{\theta_{\tau}^{\prime}}{\theta_{\tau}}\right)^{\prime}(x)-\left(\frac{\theta_{\tau}^{\prime}}{\theta_{\tau}}\right)^{\prime}(y)\right)+O(1)=\frac{1}{z}\left(\wp_{\tau}(y)-\wp_{\tau}(x)\right)+O(1)$. On a donc un pôle simple en 0 , ce qui implique que le membre de gauche est proportionnel à $\sigma_{x+y}^{\tau}$; le développement en 0 permet aussi de calculer le coefficient de proportionnalité.

L'équation (41) et le lemme 3.8 impliquent alors l'égalité

$$
\left(g_{i} \omega_{i+1}-g_{i+1} \omega_{i}\right)(\tau, z)=\left(g_{i}(0, \tau)-g_{i+1}(0, \tau)\right) \psi_{i, i+1}(\tau, z) .
$$

On a alors:

Proposition 3.9. Les $\left(\omega_{i}\right)_{i=1, \ldots, n},\left(g_{i}\right)_{i=1, \ldots, n}$ et $\left(\psi_{i, i+1}\right)_{i=1, \ldots, n-1}$ de la définition 3.6 satisfont les hypothèses de la proposition 3.1 .

En appliquant la proposition [3.1, on obtient la première partie du résultat suivant :

Théorème 3.10.

$(2 \pi \mathrm{i}) \partial_{\tau} I_{x_{1}, \ldots, x_{n}}(\tau)=\tilde{\wp}_{\tau}\left(x_{1}\right) I_{x_{2}, \ldots, x_{n}}(\tau)-\tilde{\wp}_{\tau}\left(x_{n}\right) I_{x_{1}, \ldots, x_{n-1}}(\tau)+\sum_{i=1}^{n-1}\left(\wp_{\tau}\left(x_{i+1}\right)-\wp_{\tau}\left(x_{i}\right)\right) I_{x_{1}, \ldots, x_{i}+x_{i+1}, \ldots, x_{n}}(\tau)$.

et

$$
\begin{aligned}
(2 \pi \mathrm{i}) \partial_{\tau} J_{x_{1}, \ldots, x_{n}}(\tau) & =\tilde{\wp}_{\tau}\left(x_{1}\right) J_{x_{2}, \ldots, x_{n}}(\tau)-\tilde{\wp}_{\tau}\left(x_{n}\right) J_{x_{1}, \ldots, x_{n-1}}(\tau)+\sum_{i=1}^{n-1}\left(\wp_{\tau}\left(x_{i+1}\right)-\wp_{\tau}\left(x_{i}\right)\right) J_{x_{1}, \ldots, x_{i}+x_{i+1}, \ldots, x_{n}}(\tau) \\
& -\frac{2 \pi \mathrm{i}}{\tau}\left(x_{1} \partial_{x_{1}}+\cdots+x_{n} \partial_{x_{n}}\right) J_{x_{1}, \ldots, x_{n}}(\tau) .
\end{aligned}
$$

La deuxième identité est une conséquence de la première identité et de l'identité modulaire (26), compte tenu de la relation modulaire $\tilde{\wp}_{-1 / \tau}(x)=\tau^{2} \tilde{\wp}_{\tau}(\tau x)-2 \pi \mathrm{i} \tau$, qui provient de $\wp_{-1 / \tau}(x)=$ $\tau^{2} \wp_{\tau}(\tau x)$ et de $G_{2}(-1 / \tau)=\tau^{2} G_{2}(\tau)-2 \pi \mathrm{i} \tau$ ([Se], équation (45) p. 156).

\section{RÉAlisation DE $\left\langle\delta_{2 n}, n \geq-1\right\rangle$ ET COMPARAison DE SYSTÈMES DIFFÉRENTIELS}

Comme $F=U\left(\mathfrak{f}_{2} \ominus \mathbb{C} x\right) \subset U\left(\mathfrak{f}_{2}\right)$ est un sous- $\mathfrak{f}_{2}$-module de $U\left(\mathfrak{f}_{2}\right)$, on dispose d'un sous-espace $\operatorname{Der}_{t}\left(\mathfrak{f}_{2}, F\right) \subset \operatorname{Der}_{t}\left(\mathfrak{f}_{2}, U\left(\mathfrak{f}_{2}\right)\right)=\operatorname{Der}_{t}\left(U\left(\mathfrak{f}_{2}\right)\right)$, qui est en fait une sous-algèbre de Lie. (Der ${ }_{t}$ est l'ensemble des dérivations qui envoient $t=-[x, y] \in \mathfrak{f}_{2}$ sur 0 .) D'autre part, le degré en $y$ induit une graduation de ces algèbres de Lie ; on note $\operatorname{Der}\left(\mathfrak{f}_{2}\right)_{+} \subset \operatorname{Der}\left(\mathfrak{f}_{2}\right)$ la partie de $y$-degré $>0$.

On a donc une suite d'inclusions d'algèbres de Lie

$$
\left\langle\delta_{2 n}, n \geq-1\right\rangle \subset \operatorname{Der}_{t}\left(\mathfrak{f}_{2}\right)_{+} \subset \operatorname{Der}_{t}\left(\mathfrak{f}_{2}, F\right) \subset \operatorname{Der}_{t}(F) .
$$

Le but de cette section est d'établir un isomorphisme

$$
\operatorname{Der}_{t}\left(\mathfrak{f}_{2}, F\right) \simeq \mathcal{G}_{0}
$$

entre $\operatorname{Der}_{t}\left(\mathfrak{f}_{2}, F\right)$ et une algèbre de Lie "fonctionnelle" $\mathcal{G}_{0}$ explicite, puis d'en déduire le lien entre les équations différentielles du théorème 3.10 et celles satisfaites par $A(\tau), B(\tau)$ (équations (77)). 
Pour $n \geq 1$, on pose $\mathcal{G}[n]:=\mathbb{C}\left(x_{1}, \ldots, x_{n+1}\right)$, le corps des fractions rationnelles à coefficients dans $\mathbb{C}$ en $n+1$ indéterminées $x_{1}, \ldots, x_{n+1}$. On pose

$$
\mathcal{G}:=\oplus_{n \geq 1} \mathcal{G}[n] .
$$

L'énoncé suivant est immédiat.

Proposition 4.1. $\mathcal{G}$ est munie d'une structure d'algèbre de Lie graduée donnée par

$$
[\varphi, \psi]:=\sum_{i=1}^{m+1} \varphi^{i, i+1, \ldots, i+n} \psi^{1, \ldots, i-1, i i+1 \ldots i+n, i+n+1, \ldots, n+m+1}-((\varphi, n) \leftrightarrow(\psi, m)) \in \mathcal{G}[n+m]
$$

pour $\varphi \in \mathcal{G}[n], \psi \in \mathcal{G}[m]$; on note $\varphi^{1, \ldots, n+1}:=\varphi\left(x_{1}, \ldots, x_{n+1}\right), \varphi^{12,3}:=\varphi\left(x_{1}+x_{2}, x_{3}\right)$, etc.

L'espace $F_{\infty}:=\mathbb{C}\left(x_{i}, i \in \mathbb{Z}\right)$ des fractions rationnelles en une infinité de variables est un $\mathcal{G}$-module via $\varphi * f:=\sum_{i \in \mathbb{Z}} \varphi^{i, i+1, \ldots, i+n} f^{\ldots, i-1, i i+1 \ldots i+n, i+n+1, \ldots}$.

Par l'identification de $\mathbb{C}\left[x_{1}, \ldots, x_{n}\right]$ à $\mathbb{C}\left[x_{1}, \ldots, x_{n+1}\right] /\left(x_{1}+\cdots+x_{n+1}\right)$, on obtient une action du groupe symétrique $S_{n+1}$ sur cette première algèbre (en d'autres termes, on a affaire à l'algèbre symétrique du quotient $\mathbb{C}^{n+1} / \mathbb{C}$ de la représentation naturelle par la triviale). On note $C_{n+1} \subset S_{n+1}$ le sous-groupe cyclique. L'élément $x_{1} \cdots x_{n}\left(x_{1}+\cdots+x_{n}\right)$ de $\mathbb{C}\left[x_{1}, \ldots, x_{n}\right]$ est invariant par l'action de ce groupe.

Pour $n \geq 1$, on pose

$$
\mathcal{G}_{0}[n]:=\left(\left(x_{1} \cdots x_{n}\left(x_{1}+\cdots+x_{n}\right)\right)^{-1} \mathbb{C}\left[x_{1}, \ldots, x_{n}\right]\right)^{C_{n+1}},
$$

où l'espace entre parenthèses est celui des fractions rationnelles en $x_{1}, \ldots, x_{n}$ avec dénominateur $x_{1} \cdots x_{n}\left(x_{1}+\cdots+x_{n}\right)$. On pose alors

$$
\mathcal{G}_{0}:=\oplus_{n \geq 1} \mathcal{G}_{0}[n] .
$$

Proposition 4.2. Une structure d'algèbre de Lie graduée est définie sur $\mathcal{G}_{0}$ par

$$
\begin{aligned}
{[\varphi, \psi]_{0} } & :=\sum_{i=1}^{n}\left(\varphi^{i, i+1, \ldots, i+n-1}-\varphi^{i+1, \ldots, i+n}\right) \psi^{1, \ldots, i-1, i i+1 \ldots i+n, i+n+1, \ldots, n+m} \\
& -\sum_{j=1}^{m}\left(\psi^{j, j+1, \ldots, j+m-1}-\psi^{j+1, \ldots, j+m}\right) \varphi^{1, \ldots, j-1, j j+1 \ldots j+m, j+m+1, \ldots, n+m} \\
& -\varphi^{1, \ldots, n} \psi^{n+1, \ldots, n+m}+\varphi^{m+1, \ldots, n+m} \psi^{1, \ldots, m} \in \mathcal{G}_{0}[n+m]
\end{aligned}
$$

pour $\varphi \in \mathcal{G}_{0}[n], \psi \in \mathcal{G}_{0}[m]$. L'espace $F$ a une structure de $\mathcal{G}_{0}$-module gradué par

$$
\begin{aligned}
\varphi \bullet f & :=\sum_{i=1}^{m}\left(\varphi^{i, i+1, \ldots, i+n-1}-\varphi^{i+1, \ldots, i+n}\right) f^{1, \ldots, i-1, i i+1, \ldots i+n, i+n+1, \ldots, n+m} \\
& -\varphi^{1, \ldots, n} f^{n+1, \ldots, n+m}+\varphi^{n+1, \ldots, n+m} f^{1, \ldots, m} \in F_{n+m}
\end{aligned}
$$

pour $\varphi \in \mathcal{G}_{0}[n], f \in F_{n}$. Un isomorphisme d'algèbres de Lie graduées $\operatorname{Der}_{t}\left(\mathfrak{f}_{2}, F\right) \simeq \mathcal{G}_{0}$ est donné par

$$
\operatorname{Der}_{t}\left(\mathfrak{f}_{2}, F\right)[n] \ni D \leftrightarrow \varphi\left(x_{1}, \ldots, x_{n}\right) \in \mathcal{G}_{0}[n],
$$

où $D$ est la dérivation donnée par $x \mapsto u, y \mapsto v$, où $u \in F_{n}, v \in F_{n+1}$ sont donnés par

$$
\begin{gathered}
u\left(x_{1}, \ldots, x_{n}\right)=\left(x_{1}+\cdots+x_{n}\right) \varphi\left(x_{1}, \ldots, x_{n}\right), \\
v\left(x_{1}, \ldots, x_{n+1}\right)=\left(\frac{1}{x_{1}}-\frac{1}{x_{1}+\cdots+x_{n+1}}\right) \varphi\left(x_{2}, \ldots, x_{n+1}\right)+\left(\frac{1}{x_{1}+\cdots+x_{n+1}}-\frac{1}{x_{n+1}}\right) \varphi\left(x_{1}, \ldots, x_{n}\right) .
\end{gathered}
$$


Un morphisme d'algèbres de Lie $\mathcal{G}_{0} \rightarrow \mathcal{G}$ est par ailleurs donné par

$$
\mathcal{G}_{0}[n] \ni \varphi\left(x_{1}, \ldots, x_{n}\right) \mapsto \varphi\left(x_{1}, \ldots, x_{n}\right)-\varphi\left(x_{2}, \ldots, x_{n+1}\right) \in \mathcal{G}[n] .
$$

Démonstration. Soit $D \in \operatorname{Der}_{t}\left(\mathfrak{f}_{2}, F\right)[n]$. Cet élément est déterminé par le couple $(u, v):=$ $(D(x), D(y)) \in F_{n} \times F_{n+1}$. La condition sur $(u, v)$ est

$$
\left(x_{1}+\cdots+x_{n+1}\right) v\left(x_{1}, \ldots, x_{n+1}\right)=x_{1}^{-1} u\left(x_{2}, \ldots, x_{n+1}\right)-x_{n+1}^{-1} u\left(x_{1}, \ldots, x_{n}\right)
$$

(identité dans $\left.\left(x_{1} \cdots x_{n+1}\right)^{-1} \mathbb{C}\left[x_{1}, \ldots, x_{n+1}\right]\right)$. On dispose d'une application de réduction modulo $x_{1}+\cdots+x_{n+1}$ de cet espace vers $\left(x_{1} \cdots x_{n}\left(x_{1}+\cdots+x_{n}\right)\right)^{-1} \mathbb{C}\left[x_{1}, \ldots, x_{n}\right]$. L'image de cette identité exprime alors la $C_{n+1}$-invariance de $\varphi\left(x_{1}, \ldots, x_{n}\right):=u\left(x_{1}, \ldots, x_{n}\right) /\left(x_{1}+\cdots+x_{n}\right)$. On a donc une application linéaire

$$
\operatorname{Der}_{t}\left(\mathfrak{f}_{2}, F\right)[n] \rightarrow\left(x_{1} \cdots x_{n}\left(x_{1}+\cdots+x_{n}\right)\right)^{-1} \mathbb{C}\left[x_{1}, \ldots, x_{n}\right]^{C_{n+1}},
$$

$D \mapsto \varphi$. Cette application est injective car la nullité de $u$ implique celle de $v$. Les deux dernières formules de la proposition définissent une application

$$
\left(x_{1} \cdots x_{n}\left(x_{1}+\cdots+x_{n}\right)\right)^{-1} \mathbb{C}\left[x_{1}, \ldots, x_{n}\right]^{C_{n+1}} \rightarrow F_{n} \times F_{n+1}
$$

(le pôle en $x_{1}+\cdots+x_{n+1}$ disparaissant par $C_{n+1}$-invariance), qui est en fait d'image dans $\operatorname{Der}_{t}\left(\mathfrak{f}_{2}, F\right)[n]$ et inverse à (45). On vérifie alors que le transport à $\mathcal{G}_{0}$ de la structure d'algèbre de Lie sur $\operatorname{Der}_{t}\left(\mathfrak{f}_{2}, F\right)$ et de module de $F$ sur cette algèbre de Lie est donné par les formules de l'énoncé.

On a

$$
\mathcal{G}_{0}[1]=x_{1}^{-2} \mathbb{C}\left[x_{1}^{2}\right] .
$$

Lemme 4.3. L'isomorphisme $\mathcal{G}_{0} \simeq \operatorname{Der}_{t}\left(\mathfrak{f}_{2}, F\right)$ induit la correspondance

$$
\delta_{2 n} \in \operatorname{Der}_{t}\left(\mathfrak{f}_{2}\right)_{+}[1] \subset \operatorname{Der}_{t}\left(\mathfrak{f}_{2}, F\right)[1] \leftrightarrow \mathcal{G}_{0}[1] \ni x_{1}^{2 n} .
$$

Démonstration. La dérivation correspondant à $x_{1}^{2 n}$ est une dérivation de $\operatorname{Der}_{t}\left(\mathfrak{f}_{2}, F\right)$ telle que $x \mapsto u=x_{1}^{2 n+1} \leftrightarrow\left[x^{2 n+2} y\right]$. Comme l'application $\operatorname{Der}_{t}\left(\mathfrak{f}_{2}, F\right) \rightarrow F, D \mapsto D(x)$ est injective, cette dérivation coincide avec la dérivation $\delta_{2 n}$ définie en section 1.2 .3 .

Rappelons par ailleurs la correspondance

$$
e^{\mathrm{i} \pi t} A(\tau) \in U\left(\mathfrak{f}_{2} \ominus \mathbb{C} x\right) \leftrightarrow F \ni\left((-1)^{n} I_{x_{n}, \ldots, x_{1}}(\tau)\right)_{n \geq 0}=: \tilde{I}(\tau) .
$$

(section 2.4). D'après (7) et l'invariance de $t$ sous les $\delta_{2 n}, n \geq-1, e^{\mathrm{i} \pi t} A(\tau)$ satisfait l'équation différentielle

$$
2 \pi \mathrm{i} \partial_{\tau}\left(e^{\mathrm{i} \pi t} A(\tau)\right)=-\left(\sum_{n \geq-1}(2 n+1) G_{2 n+2}(\tau) \delta_{2 n}\right)\left(e^{\mathrm{i} \pi t} A(\tau)\right)
$$

L'image de cette équation différentielle sous l'isomorphisme $U\left(\mathfrak{f}_{2} \ominus \mathbb{C} x\right) \simeq F$ donne

$$
2 \pi \text { i } \partial_{\tau} \tilde{I}(\tau)=-\left(\sum_{n \geq-1}(2 n+1) G_{2 n+2}(\tau) x_{1}^{2 n}\right) \bullet \tilde{I}(\tau)=-\tilde{\wp}_{\tau}\left(x_{1}\right) \bullet \tilde{I}(\tau),
$$

donc si $I(\tau):=\left(I_{x_{1}, \ldots, x_{n}}(\tau)\right)_{n \geq 0}$, alors $2 \pi$ i $\partial_{\tau} I(\tau)=-\tilde{\wp}_{\tau}\left(x_{1}\right) \bullet I(\tau)$, c'est-à-dire que pour chaque $n$

$$
2 \pi \mathrm{i} \partial_{\tau} I_{x_{1}, \ldots, x_{n}}(\tau)=-\tilde{\wp}_{\tau}\left(x_{1}\right) \bullet I_{x_{1}, \ldots, x_{n-1}}(\tau) .
$$

Compte tenu de la formule (44) pour l'action de $\mathcal{G}_{0}$ sur $F$, on retrouve ainsi la première équation différentielle du théorème 3.10 .

De même, $e^{-\mathrm{i} \pi t} B(\tau)$ satisfait l'équation différentielle

$$
2 \pi \mathrm{i} \partial_{\tau}\left(e^{-\mathrm{i} \pi t} B(\tau)\right)=-\left(\sum_{n \geq 1}(2 n+1) G_{2 n+2}(\tau) \delta_{2 n}\right)\left(e^{-\mathrm{i} \pi t} B(\tau)\right) .
$$


Soit $\tilde{B}(\tau):=\exp \left(\frac{2 \pi \mathrm{i}}{\tau} e_{+}\right)\left(e^{-\mathrm{i} \pi t} B(\tau)\right)$ (voir section 2.4), on en déduit

$$
2 \pi \mathrm{i} \partial_{\tau} \tilde{B}(\tau)=-\left(\frac{2 \pi \mathrm{i}}{\tau} h+\sum_{n \geq-1}(2 n+1) G_{2 n+2}(\tau) \delta_{2 n}\right)(\tilde{B}(\tau)),
$$

où $h:=\left[e_{+}, \delta\right]$ est la dérivation de $\mathfrak{f}_{2}$ donnée $\operatorname{par}(x, y) \mapsto(x,-y)$, compte tenu de $\left[e_{+}, \delta_{2 n}\right]=0$ si $n \geq 0$ et $\frac{1}{2}\left[e_{+},\left[e_{+}, \delta_{-2}\right]\right]+e_{+}=0$.

On a la correspondance

$$
U\left(\mathfrak{f}_{2} \ominus \mathbb{C} x\right) \ni \tilde{B}(\tau) \leftrightarrow\left((-1)^{n} J_{x_{n}, \ldots, x_{1}}(\tau)\right)=: \tilde{J}(\tau) \in F,
$$

par ailleurs la dérivation $h$ se transporte sous cette correspondance en la dérivation de $F=\oplus_{n \geq 0} F_{n}$ de degré zéro, opérant sur $F_{n}$ comme $\xi:=\sum_{i=1}^{n} x_{i} \partial_{x_{i}}$. On en déduit

$$
2 \pi \mathrm{i} \partial_{\tau} \tilde{J}(\tau)=-\left(\frac{2 \pi \mathrm{i}}{\tau} \xi+\tilde{\wp}_{\tau}\left(x_{1}\right) \bullet\right) \tilde{J}(\tau)
$$

donc $J(\tau):=\left(J_{x_{1}, \ldots, x_{n}}(\tau)\right)_{n \geq 0}$ satisfait la même équation différentielle, donc

$$
2 \pi \mathrm{i} \partial_{\tau} J_{x_{1}, \ldots, x_{n}}(\tau)=-\frac{2 \pi \mathrm{i}}{\tau}\left(\sum_{i=1}^{n} x_{i} \partial_{x_{i}}\right) J_{x_{1}, \ldots, x_{n}}(\tau)-\tilde{\wp}_{\tau}\left(x_{1}\right) \bullet J_{x_{1}, \ldots, x_{n-1}}(\tau),
$$

ce qui permet de retrouver la deuxième équation différentielle du théorème 3.10 .

\section{DÉveloppement ASymptotique des ANAlogues Elliptiques Des nOMBRES MultizÉtas}

Dans cette section, nous utilisons les équations différentielles satisfaites par les fonctions $A(\tau)$ et $B(\tau)$ (équations (7)) et leur comportement à l'infini ((8)), (9)) pour en obtenir un développement asymptotique en $\tau \rightarrow \mathrm{i} \infty$. Nous en déduisons la forme du développement asymptotique des fonctions $I_{\underline{d}}(\tau), J_{\underline{d}}(\tau)$ dans cette région.

5.1. Développement de $g(\tau)$. Soit $\mathfrak{G}$ la complétion de l'algèbre de Lie $\left\langle\delta_{2 n}, n \geq-1\right\rangle \subset \operatorname{Der}_{t}\left(\mathfrak{f}_{2}\right)$ pour le bidegré en $(x, y)$; on a $\left|\delta_{2 n}\right|=(2 n+1,1)$. Soit $G:=\exp (\mathfrak{G}) \subset$ Aut $_{t}\left(\hat{\mathfrak{f}}_{2}\right)$ le groupe de Lie correspondant.

Proposition 5.1. Il existe une unique fonction $g(\tau): \mathfrak{H} \rightarrow G$, telle que

$$
2 \pi \mathrm{i} \partial_{\tau} g(\tau)=-\left(\sum_{n \geq-1}(2 n+1) G_{2 n+2}(\tau) \delta_{2 n}\right) g(\tau)
$$

et $g(\tau) \simeq e^{\frac{-1}{2 \pi \mathrm{i}}\left(\delta_{-2}+\sum_{n \geq 0}(2 n+1) \cdot 2 \zeta(2 n+2) \delta_{2 n}\right) \tau}=e^{D_{0} \tau}$ en $\tau \rightarrow \mathrm{i} \infty$. Il existe une collection $\left(h_{k}\right)_{k \geq 0}$, avec $h_{0}=1$, telle que $g(\tau)$ a le développement asymptotique

$$
g(\tau) \simeq \sum_{k, n \geq 0} \frac{1}{n !} h_{k} D_{0}^{n} \tau^{n} e^{2 \pi \mathrm{i} k \tau}
$$

en $\tau \rightarrow \mathrm{i} \infty$.

Démonstration. Posons $D(\tau):=\frac{-1}{2 \pi \mathrm{i}} \sum_{n \geq-1}(2 n+1) G_{2 n+2}(\tau) \delta_{2 n}$. Posons si $m \geq 1, g_{2 m}(n):=$ $\frac{2(2 \pi \mathrm{i})^{2 m}}{(2 m-1) !} \sigma_{2 m-1}(n)$ (où $\sigma_{k}(n)=\sum_{d \mid n} d^{k}$ ) si $n>0$, et $g_{2 m}(0):=2 \zeta(2 m)$; et posons $g_{0}(n)=0$ si $n>0$, et $g_{0}(0)=-1$. Alors $G_{2 m}(\tau)=\sum_{n \geq 0} g_{2 m}(n) e^{2 \pi \mathrm{i} n \tau}$ et

$$
D(\tau)=\sum_{m \geq 0} D_{m} e^{2 \pi \mathrm{i} m \tau} \text {, où } D_{m}:=\frac{-1}{2 \pi \mathrm{i}} \sum_{n \geq-1}(2 n+1) g_{2 n+2}(m) \delta_{2 n} .
$$


Comme $D_{0}$ est de $y$-degré $1,2 \pi \mathrm{i} m-$ ad $D_{0}$ est inversible dans $\operatorname{End}(U \mathfrak{G})$ si $m>0$. Définissons $\left(h_{m}\right)_{m \geq 0}$ par $h_{0}:=1$,

$$
h_{m}:=\left(2 \pi \mathrm{i} m-\operatorname{ad} D_{0}\right)^{-1}\left(\sum_{\substack{m^{\prime}+m^{\prime \prime}=m \\ m^{\prime}>0}} D_{m^{\prime}} h_{m^{\prime \prime}}\right) \text { si } m>0 .
$$

Alors $h(\tau):=\sum_{m \geq 0} h_{m} e^{2 \pi \text { i } m \tau}$ est une solution formelle de $\partial_{\tau} h(\tau)=D(\tau) h(\tau)-h(\tau) D_{0}$, qui est l'équation différentielle satisfaite par $g(\tau) e^{-D_{0} \tau}$; cette fonction admet donc $h(\tau)$ comme développement asymptotique.

\subsection{Développements de $A(\tau), B(\tau)$. Posons}

$$
A_{\infty}:=\Phi(\tilde{y}, t) e^{2 \pi \mathrm{i} \tilde{y}} \Phi(\tilde{y}, t)^{-1}, \quad \underline{B}(\tau):=e^{\mathrm{i} \pi t} \Phi(-\tilde{y}-t, t) e^{2 \pi \mathrm{i} x} e^{2 \pi \mathrm{i} \tilde{y} \tau} \Phi(\tilde{y}, t)^{-1}, \quad B_{\infty}:=B[0] .
$$

D'après [CEE], on a

$$
A_{\infty}=e^{\tau D_{0}}\left(A_{\infty}\right), \quad \underline{B}(\tau)=e^{\tau D_{0}}\left(B_{\infty}\right) .
$$

Posons $h(\tau):=g(\tau) e^{-\tau D_{0}}$, alors d'après la proposition 5.1, $h(\tau)$ admet le développement asymptotique $h(\tau) \simeq 1+\sum_{m>0} h_{m} e^{2 \pi \mathrm{i} m \tau}$.

On a alors $A(\tau)=g(\tau)\left(A_{\infty}\right)=h(\tau)\left(A_{\infty}\right)$ donc $A(\tau)$ admet le développement asymptotique

$$
A(\tau) \simeq \sum_{m \geq 0} e^{2 \pi \mathrm{i} m \tau} h_{m}\left(A_{\infty}\right)
$$

et $B(\tau)=g(\tau)\left(B_{\infty}\right)=h(\tau)(\underline{B}(\tau))$, donc $\tilde{B}(\tau)$ admet le développement asymptotique

$$
\tilde{B}(\tau) \simeq \exp \left(-\frac{2 \pi \mathrm{i}}{\tau} e_{+}\right) h(\tau)(\underline{B}(\tau))
$$

5.3. Développements de $I_{\underline{d}}(\tau), J_{\underline{d}}(\tau)$. Soit $\mathbf{k}_{M Z V} \subset \mathbb{C}$ le $\mathbb{Q}$-sous-anneau engendré par les multizétas. L'associateur $\Phi$ étant à coefficients dans $\mathbf{k}_{M Z V}$, on déduit de (5.2) et (5.2) :

Proposition 5.2. Les fonctions $I_{\underline{d}}(\tau), J_{\underline{d}}(\tau)$ admettent les développements asymptotiques

$$
I_{\underline{d}}(\tau) \simeq \sum_{n \geq 0} I_{\underline{d}, n} e^{2 \pi \mathrm{i} n \tau}, \quad J_{\underline{d}}(\tau) \simeq \sum_{n \geq 0} \sum_{s \in \mathbb{Z}} J_{\underline{d}, n, s} \tau^{s} e^{2 \pi \mathrm{i} n \tau},
$$

dans lesquels les coefficients sont dans $\mathbf{k}_{M Z V}[2 \pi \mathrm{i}]$. Dans la deuxième série, la deuxième somme $\sum_{s}$ est finie pour tout $n \geq 0$.

\section{BIBLIOGRAPHIE}

[BL] F. Brown, A. Levin, Multiple elliptic polylogarithms, preprint arXiv:1110:6917 (2011, revised in 2013).

[CEE] D. Calaque, B. Enriquez, B. Etingof, Universal KZB equations: the elliptic case. Algebra, arithmetic, and geometry: in honor of Yu. I. Manin. Vol. I, 165-266, Progr. Math., 269, Birkhäuser Boston, Inc., Boston, MA, 2009.

[Dr] V. Drinfeld, On quasitriangular quasi-Hopf algebras and a group closely connected with Gal $(\overline{\mathbb{Q}} / \mathbb{Q})$, Leningrad Math. J. 2 (1991), 829-860.

[En1] B. Enriquez, Quasi-reflection algebras and cyclotomic associators, Selecta Math. (N.S.) 13 (2007), no. 3, 391463.

[En2] B. Enriquez, Elliptic associators, Selecta Math. (N.S.) 20 (2014), no. 2, 491-584.

$[\mathrm{Fu}]$ H. Furusho, Double shuffle relation for associators. Ann. of Math. (2) 174 (2011), no. 1, 341-360.

$[\mathrm{HM}]$ R. Hain, M. Matsumoto, exposé au workshop "Multizeta values, modular forms and elliptic motives", Heilbronn Institute of Mathematical Research, Bristol (2011).

[LM] T.T.Q. Le, J. Murakami, Kontsevich's integral for the Kauffman polynomial. Nagoya Math. J. 142 (1996), 39-65.

[LR] A. Levin, G. Racinet, Towards multiple elliptic polylogarithms, arXiv:math/0703237, preprint (2007).

[Po] A. Polishchuk, Abelian varieties, theta functions and the Fourier transform. Cambridge Tracts in Mathematics, 153. Cambridge University Press, Cambridge, 2003.

[Pk] A. Pollack, Relations between derivations arising from modular forms, thèse de Ph.D., Duke University (2009). 
[Se] J.-P. Serre, Cours d'arithmétique. Collection SUP : "Le Mathématicien", 2. Presses Universitaires de France, Paris 1970 .

[Za] D. Zagier, Values of zeta functions and their applications. First European Congress of Mathematics, Vol. II (Paris, 1992), 497-512, Progr. Math., 120, Birkhäuser, Basel, 1994.

IRMA (CNRS), Université de Strasbourg, 7 rue René Descartes, F-67084 Strasbourg, France E-mail address: b.enriquez@math.unistra.fr 